\title{
Fifteen new species of Sathytes Westwood from China (Coleoptera, Staphylinidae, Pselaphinae), with an updated checklist of world species
}

\author{
Zi-Wei YIN ${ }^{1, *} \&$ Qi SHEN ${ }^{2}$ \\ ${ }^{1,2}$ Laboratory of Systematic Entomology, College of Life Sciences, Shanghai Normal University, \\ 100 Guilin Road, Xuhui District, Shanghai 200234, China. \\ "Corresponding author: pselaphinae@gmail.com \\ 2Email: 2974259092@qq.com \\ ${ }^{1}$ urn:1sid:zoobank.org:author:C515106C-5782-4471-AE11-483B49606A67 \\ ${ }^{2}$ urn:lsid:zoobank.org:author:195E5F86-BD32-45F2-BF46-014394EF380F
}

\begin{abstract}
The Chinese fauna of the pselaphine genus Sathytes Westwood (Batrisitae: Batrisini) currently includes 20 species. In this paper, 15 new species from various provinces of the country are described: S. alpicola sp. nov. (Xizang), S. australis sp. nov. (Guangdong, Guangxi), S. chayuensis sp. nov. (Xizang), S. chengzhifeii sp. nov. (Yunnan), S. huapingensis sp. nov. (Guangxi), S. linzhiensis sp. nov. (Xizang), S. maoershanus sp. nov. (Guangxi), S. nujiangensis sp. nov. (Yunnan), S. panzhaohuii sp. nov. (Xizang), S. shennong sp. nov. (Hubei), S. tianquanus sp. nov. (Sichuan), S. transversus sp. nov. (Xizang), S. valentulus sp. nov. (Guangxi), S.xingdoumontis sp. nov. (Hubei) and S. xizangensis sp. nov. (Xizang). New collection records are provided for S. longitrabis Yin \& Li, 2012, S. tangliangi Yin \& Li, 2012 and S. yunnanicus Yin \& Li, 2012. Maps showing the distribution of the genus in China, and an updated checklist of the world species are provided.
\end{abstract}

Keywords. Taxonomy, Sathytes, biodiversity, new species, China.

Yin Z.-W. \& Shen Q. 2020. Fifteen new species of Sathytes Westwood from China (Coleoptera, Staphylinidae, Pselaphinae), with an updated checklist of world species. European Journal of Taxonomy 722: 37-74. https://doi.org/10.5852/ejt.2020.722.1133

\section{Introduction}

The ant-loving beetle genus Sathytes Westwood, 1870 of the tribe Batrisini Reitter, 1882 currently comprises 42 described species distributed in the southern Palaearctic and Oriental regions (Shen \& Yin 2020). Members of this group are highly recognizable by their coarse cuticle, while species identifications within the genus heavily rely on careful examination of the form of the male antennal clubs, and distributions. The aedeagus, which is normally of great diagnostic significance in beetles, is greatly simplified in Sathytes to form a flattened, plate-like median lobe, thus is less useful in species identification. The biology of Sathytes is largely unknown. According to existing records, most species 
were collected from sifted leaf litter samples, such that they are presumed to be predators of smaller invertebrates.

Since the first revision of Sathytes (Löbl 1979), the known world fauna has increased from 18 species (with an addition of three unnamed species from Pahang, Java and Taiwan) to 42, with 20 additional species described from China (Yin et al. 2012; Shen \& Yin 2020), and four species from East Malaysia (Shen \& Yin 2019). Like many other pselaphine groups, the diversity of Sathytes in Asia remains underexplored, especially in those areas where no proper sampling has been pursued. A great number of undescribed species are expected to be found after future fieldwork has been conducted in these areas.

In the present paper, 15 new species of Sathytes are described from various provinces of China, with their morphology compared to related congeners. Additionally, new collection records are given for three previously known species. The habitus and major diagnostic characters of each new species are illustrated; maps showing the distribution of the genus in China as well as an updated checklist of the world species is provided.

\section{Material and methods}

The material treated in this study is housed in the Institute of Zoology, Chinese Academy of Sciences (IZCAS), and the Insect Collection of Shanghai Normal University (SNUC). The label data of the material are quoted verbatim, except for a few modifcations in format required by the journal.

Dissected parts were preserved in Euparal on plastic slides that were placed on the same pin as the specimens. The habitus images were taken using a Canon 5D Mark III camera in conjunction with a Canon MP-E $65 \mathrm{~mm}$ f/2.8 1-5× Macro Lens, and a Canon MT-24EX Macro Twin Lite Flash was used as the light source. Images of morphological details were produced using a Canon G9 camera mounted to an Olympus CX31 microscope under transmitted light. Zerene Stacker (ver. 1.04) was used for image stacking. All images were optimized and grouped into plates using Adobe Photoshop CS5 Extended.

\section{Abbreviations used in measurements}

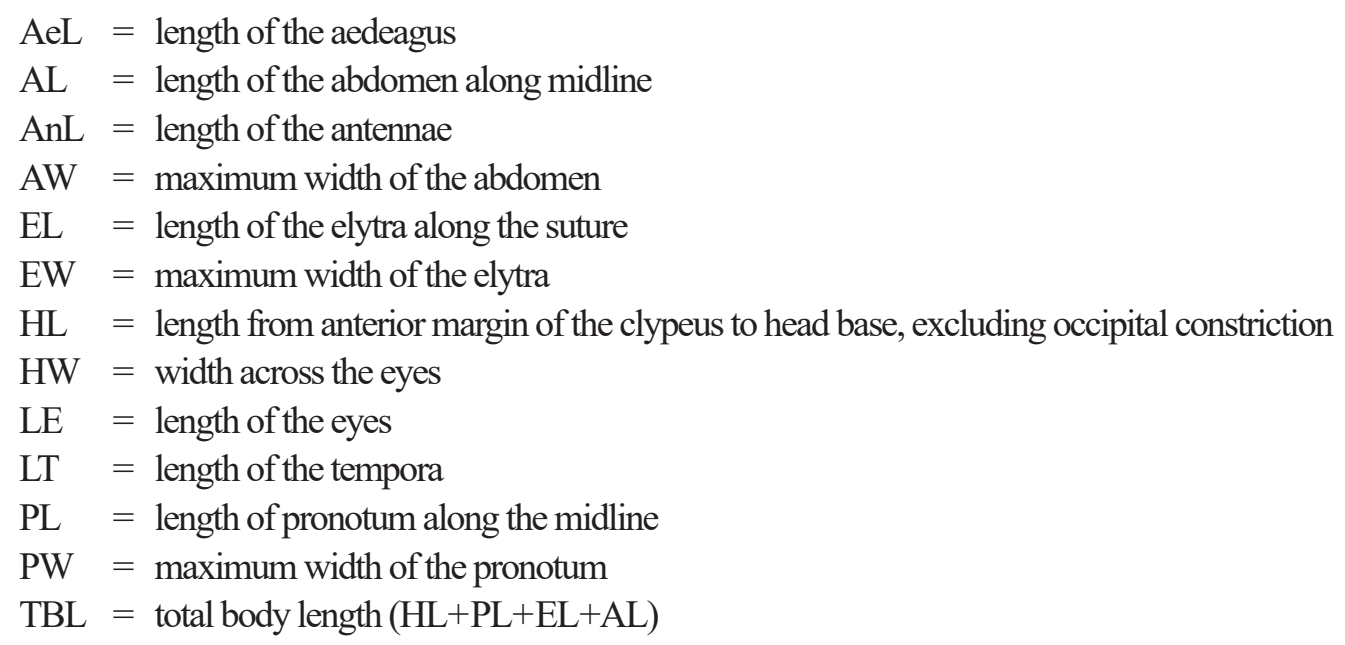




\title{
Results
}

\section{Taxonomic treatments}

\author{
Class Insecta Linnaeus, 1758 \\ Order Coleoptera Linnaeus, 1758 \\ Family Staphylinidae Latreille, 1802 \\ Subfamily Pselaphinae Latreille, 1802 \\ Genus Sathytes Westwood, 1870 \\ Sathytes alpicola sp. nov. \\ urn:1sid:zoobank.org:act:71682078-E347-49C1-B2AF-C13A61694281
}

Fig. 1

\section{Differential diagnosis}

The male of S. alpicola sp. nov. is morphologically similar to that of S. grandis Löbl, 1979, S. montanus Löbl, 1979, S. magnus Yin \& Li, 2012, S. chayuensis sp. nov. and S. xizangensis sp. nov. from the Himalayan region, and S. tangliangi Yin \& Li, 2012 from Yunnan, southwestern China in the relatively large body size $(2.53-3.36 \mathrm{~mm})$ and the dense golden pubescence that covers the dorsal surface of the body. The difference between $S$. alpicola sp. nov. and a few other species, e.g., S. xizangensis sp. nov., is subtle. However, the male of $S$. alpicola sp. nov. can be separated from that of S. xizangensis sp. nov. and all related species by the unique form of the antennomere 9 (slightly concave on mesal margin) and the apophysis on it (rounded at the apex).

\section{Etymology}

The specific name indicates its distribution in high-altitude mountains.

Type material (4 specimens)

\section{Holotype}

CHINA - O'; Xizang, Motuo County (墨脱县), near $80 \mathrm{~K}$; 2941'09" N, 95³0'10" E; alt. 2330 m; 9 Jul. 2018; Cheng, Peng and Shen leg.; mixed leaf litter, sifted; SNUC.

\section{Paratypes}

CHINA $• 3$ 우; same collection data as for holotype; SNUC.

\section{Description}

\section{Male}

MeAsurements. AeL $=0.27 \mathrm{~mm} ; \mathrm{AnL}=1.51 \mathrm{~mm} ; \mathrm{AL}=0.78 \mathrm{~mm} ; \mathrm{AW}=0.94 \mathrm{~mm} ; \mathrm{TBL}=2.96 \mathrm{~mm}$; $\mathrm{EL}=0.87 \mathrm{~mm} ; \mathrm{EW}=1.90 \mathrm{~mm} ; \mathrm{HL}=0.67 \mathrm{~mm} ; \mathrm{HW}=0.57 \mathrm{~mm} ; \mathrm{LE}=0.18 \mathrm{~mm} ; \mathrm{LT}=0.23 \mathrm{~mm} ; \mathrm{PL}=$ $0.64 \mathrm{~mm} ; \mathrm{PW}=0.65 \mathrm{~mm}$.

Body (Fig. 1A) reddish-brown, mouthparts and tarsi paler. Head distinctly longer than wide. Each eye composed of about 22 facets; LE: $\mathrm{LT}=0.8$. Antennal clubs (Fig. 1B) formed by antennomeres 9-11; inner margin of antennomere 9 (Fig. 1C) slightly concave on mesal margin, semi-membranous apophysis at basal $1 / 3$ on mesal surface; antennomere 10 strongly transverse, each expanded and rounded at apex; antennomere 11 (Fig. 1B) about 1.7 times as long as wide, each with single basal protuberance covered with thin tuft of setae at apex (Fig. 1D). Pronotum about as long as wide. Elytra strongly transverse. Abdomen widest at tergite 1 (IV) and narrowing apically. Aedeagus (Fig. 1E) slightly asymmetrical, slightly broadened apically. 


\section{Female}

Measurements. AnL $=1.21-1.22 \mathrm{~mm} ; \mathrm{AL}=0.87-0.88 \mathrm{~mm} ; \mathrm{AW}=0.89-0.90 \mathrm{~mm} ; \mathrm{TBL}=2.82-2.88 \mathrm{~mm}$; $\mathrm{EL}=0.74-0.76 \mathrm{~mm} ; \mathrm{EW}=0.95-0.96 \mathrm{~mm} ; \mathrm{HL}=0.59-0.60 \mathrm{~mm} ; \mathrm{HW}=0.51-0.52 \mathrm{~mm} ; \mathrm{LE}=0.14 \mathrm{~mm}$; $\mathrm{LT}=0.19 \mathrm{~mm} ; \mathrm{PL}=0.62-0.64 \mathrm{~mm} ; \mathrm{PW}=0.58-0.59 \mathrm{~mm}$.

Slightly smaller than male in size. Each eye composed of about 14 facets; LE: LT = 1.6. Antennal clubs lacking modifications.

\section{Distribution}

China: Xizang (Tibet).
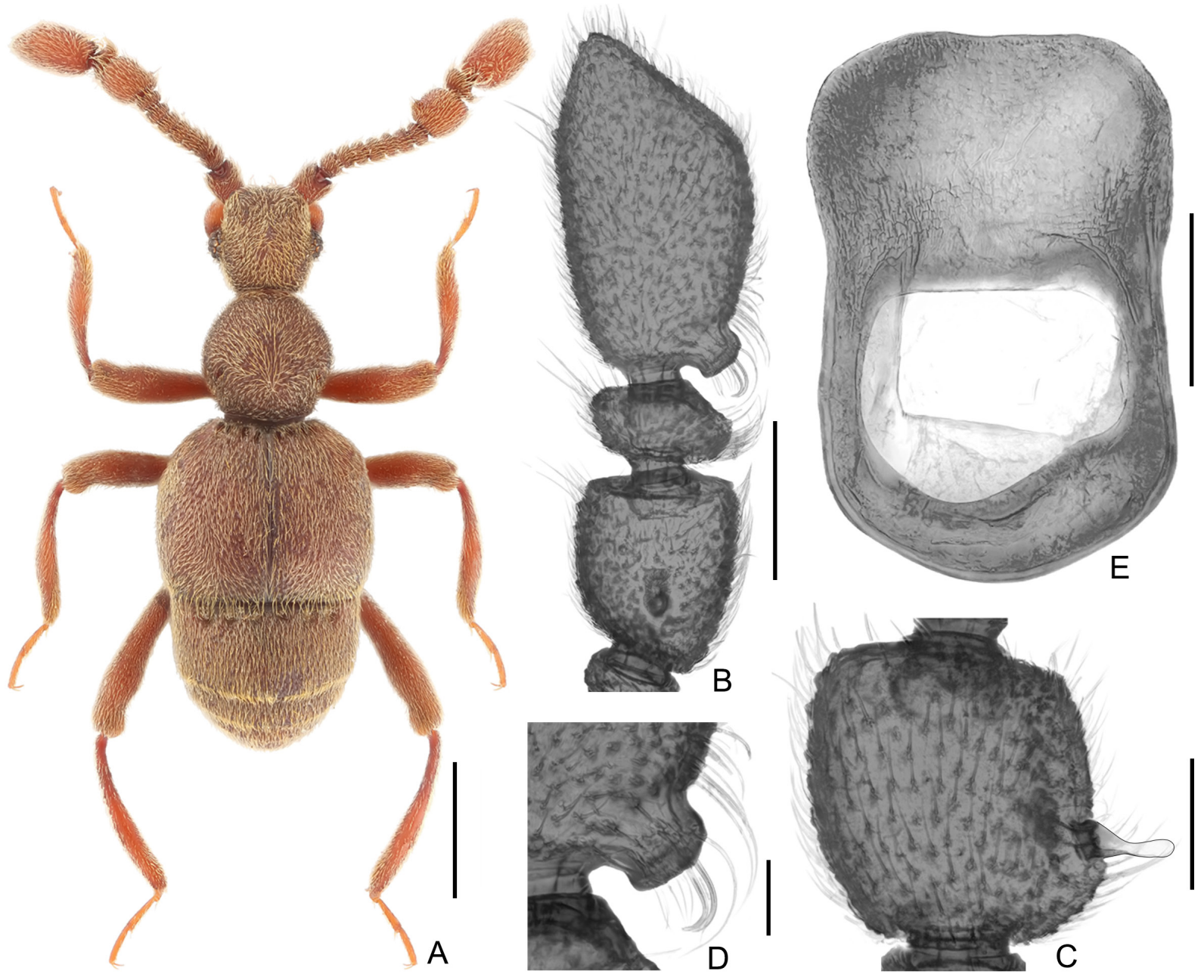

Fig. 1. Sathytes alpicola sp. nov., O. A. Dorsal habitus. B. Antennal club. C. Antennomere 9. D. Modification of antennomere 11, enlarged. E. Aedeagus, ventral view. Scale bars: A $=0.5 \mathrm{~mm}$; $\mathrm{B}=$ $0.2 \mathrm{~mm} ; \mathrm{C}, \mathrm{E}=0.1 \mathrm{~mm}$; $\mathrm{D}=0.05 \mathrm{~mm}$. 
Sathytes australis sp. nov.

urn:1sid:zoobank.org:act:074C636B-E7FE-4032-9E2B-781DB0DDA329

Fig. 2

\section{Differential diagnosis}

The male of S. australis sp. nov. shares with S. similis Shen \& Yin, 2020 (China: Zhejiang, Jiangxi) the similar body size and structure of the antennal clubs. These two species can be separated only by the following subtle differences: in S. australis sp. nov., the eyes are slightly larger, the dorsal margin of antennomere 11 is more convex, the upper (subbasal) protuberance of antennomere 11 is smaller, antennomere 9 in full view (when full widths are visible) is slightly more transverse and the apex of the aedeagus is narrower, and by their different distributions.

\section{Etymology}

The specific name indicates the distribution of the new species in southern China.

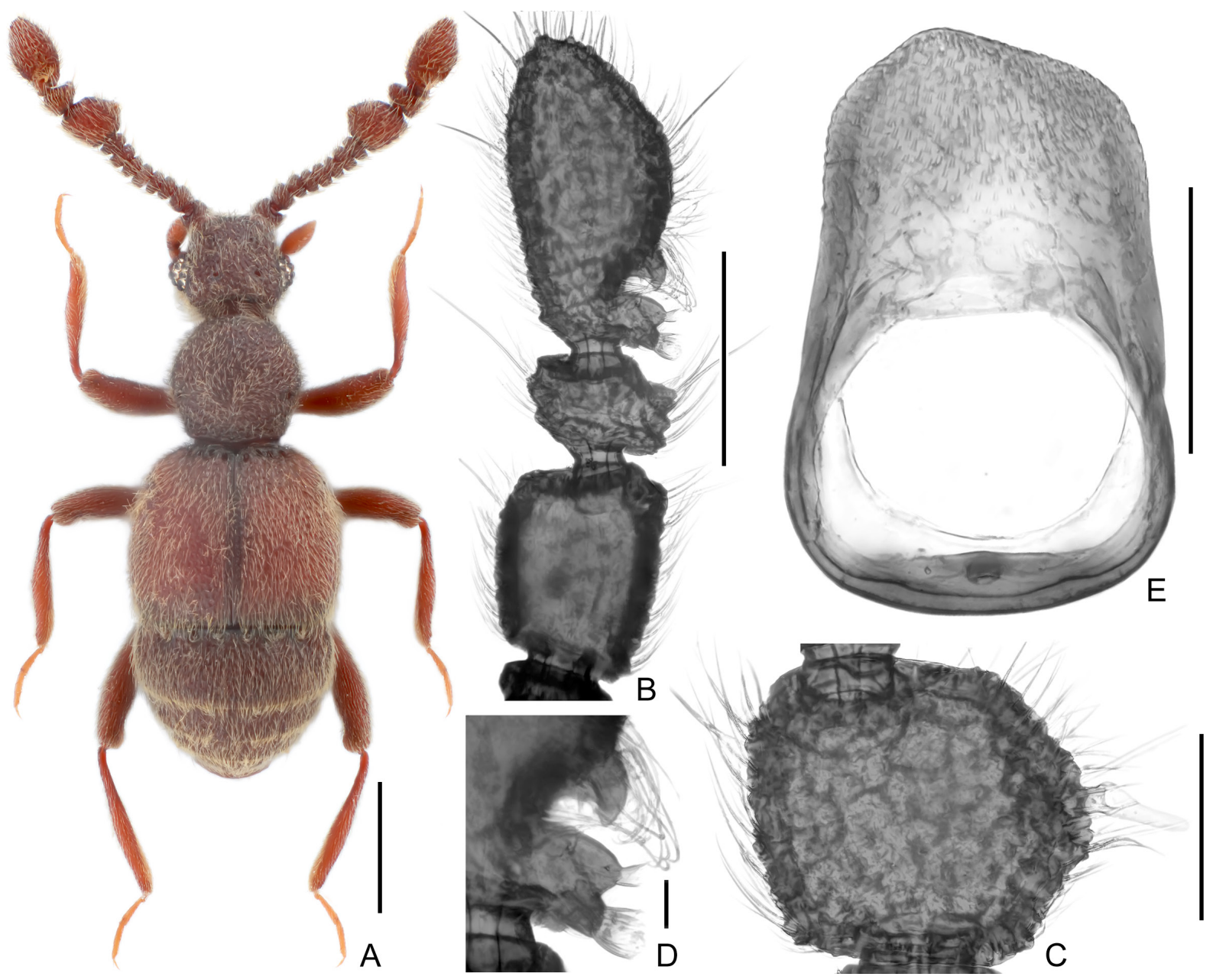

Fig. 2. Sathytes australis sp. nov., ô (population of Maoershan). A. Dorsal habitus. B. Antennal club. C. Antennomere 9. D. Modification of antennomere 11, enlarged. E. Aedeagus, ventral view. Scale bars: $\mathrm{A}=0.5 \mathrm{~mm} ; \mathrm{B}=0.2 \mathrm{~mm} ; \mathrm{C}, \mathrm{E}=0.1 \mathrm{~mm} ; \mathrm{D}=0.05 \mathrm{~mm}$. 
Type material (4 specimens)

\section{Holotype}

CHINA - ○' Guangxi, Guilin City (桂林市), Xing'an County (兴安县), Maoer shan (猫儿山); $25^{\circ} 51^{\prime} 28^{\prime \prime}$ N, $110^{\circ} 29^{\prime} 04^{\prime \prime}$ E; alt. 450-650 m; 25 Jul. 2012; Hu and Song leg.; mixed leaf litter, sifted; SNUC.

\section{Paratypes}

CHINA - 1 ' ; same collection data as for holotype; $25^{\circ} 53^{\prime} 15^{\prime \prime} \mathrm{N}, 110^{\circ} 25^{\prime} 47^{\prime \prime} \mathrm{E}$; alt. $2030 \mathrm{~m}$; $30 \mathrm{Jul}$. 2014; Peng, Song, Yu and Yan leg.; beech forest, mixed leaf litter, humus, sifted; SNUC • 1 गे; same collection data as for holotype; Gaozhai (高寨村); 10 Aug. 2006; Jian-Hua Huang leg.; SNUC • 1 j ; Guangdong Province, Ruyuan Hsien (乳源县), Nanling N.R. (南岭自然保护区), Walkway; 24 ${ }^{\circ} 5^{\prime} 57^{\prime \prime}$ N, 11300'18" E; alt. 1220 m; 28 Apr. 2015; Peng and Zhou leg.; mixed forest, leaf litter, wood, sifted; SNUC.

\section{Description}

\section{Male}

MeAsurements. AeL $=0.22 \mathrm{~mm}$; $\mathrm{AnL}=0.95-1.08 \mathrm{~mm} ; \mathrm{AL}=0.56-0.60 \mathrm{~mm} ; \mathrm{AW}=0.71-0.74 \mathrm{~mm}$; $\mathrm{TBL}=2.07-2.18 \mathrm{~mm} ; \mathrm{EL}=0.67-0.68 \mathrm{~mm} ; \mathrm{EW}=0.78-0.79 \mathrm{~mm} ; \mathrm{HL}=0.37-0.40 \mathrm{~mm} ; \mathrm{HW}=0.46-$ $0.47 \mathrm{~mm} ; \mathrm{LE}=0.16 \mathrm{~mm} ; \mathrm{LT}=0.09 \mathrm{~mm} ; \mathrm{PL}=0.47-0.50 \mathrm{~mm} ; \mathrm{PW}=0.47-0.49 \mathrm{~mm}$.

Body (Fig. 2A) dark reddish-brown, mouthparts and tarsi paler. Head much wider than long. Each eye composed of about 20 facets; LE: $\mathrm{LT}=1.8$. Antennal clubs (Fig. 2B) formed by antennomeres 9-11; antennomere 9 (Fig. 2C) slightly transverse, inner margin moderately expanded mesally, with single semi-membranous apophysis at apex of expansion; antennomere 10 strongly transverse, obconical; antennomere 11 (Fig. 2B) about 1.8 times as long as wide, each with two basal protuberances, more apical one short, rounded at apex, more basal one covered with two tufts of setae at apex (Fig. 2D). Pronotum about as long as wide. Elytra strongly transverse. Abdomen widest at tergite 1 (IV) and narrowing apically. Aedeagus (Fig. 2E) slightly asymmetrical at apex.

\section{Female}

Unknown.

\section{Distribution}

China: Guangdong, Guangxi.

Sathytes chayuensis sp. nov.

urn:lsid:zoobank.org:act:B95327A9-4E88-4DA8-818F-319126D7340A

Fig. 3

\section{Differential diagnosis}

The male of $S$. chayuensis sp. nov. can be separated from that of all congeners by a single basal protuberance on the terminal antennomere and by the remarkably elongate antennomere 11 .

\section{Etymology}

The new species is named after Chayu County, where the type locality of this species is situated.

Type material (4 specimens)

\section{Holotype}

CHINA - J; Xizang, Chayu Co. (察隅县), Xiachayu To. (下察隅镇), Talin Bridge (塔林桥); $28.53340539^{\circ} \mathrm{N}, 96.97800188^{\circ}$ E; alt. 1541 m; 30 Jul. 2019; Liang Lü leg.; SNUC. 


\section{Paratypes}

CHINA $\bullet 2 \widehat{\partial}, 1$; ; same collection data as for holotype; SNUC.

\section{Description}

\section{Male}

Measurements. AeL $=0.33 \mathrm{~mm} ; \mathrm{AnL}=1.53-1.56 \mathrm{~mm} ; \mathrm{AL}=0.76-0.79 \mathrm{~mm} ; \mathrm{AW}=0.97-1.01 \mathrm{~mm}$; $\mathrm{TBL}=2.80-2.99 \mathrm{~mm} ; \mathrm{EL}=0.93-0.96 \mathrm{~mm} ; \mathrm{EW}=1.10-1.15 \mathrm{~mm} ; \mathrm{HL}=0.58-0.61 \mathrm{~mm} ; \mathrm{HW}=0.55-$ $0.57 \mathrm{~mm} ; \mathrm{LE}=0.21 \mathrm{~mm} ; \mathrm{LT}=0.21 \mathrm{~mm} ; \mathrm{PL}=0.66-0.70 \mathrm{~mm} ; \mathrm{PW}=0.64-0.68 \mathrm{~mm}$.

Body (Fig. 3A) reddish-brown, mouthparts and tarsi paler. Head wider than long. Each eye composed of about 25 facets; LE: LT = 1. Antennal clubs (Fig. 3B) formed by antennomeres 9-11; antennomere 9 (Fig. 3C) slightly transverse, inner margin angularly expanded mesally, each with single semimembranous apophysis anterior to apex of expansion; antennomere 10 strongly transverse, obconical; antennomere 11 (Fig. 3B) about 2.1 times as long as wide, each with single apically concave basal protuberance covered with tuft of elongate setae at apex (Fig. 3D). Pronotum about as long as wide.
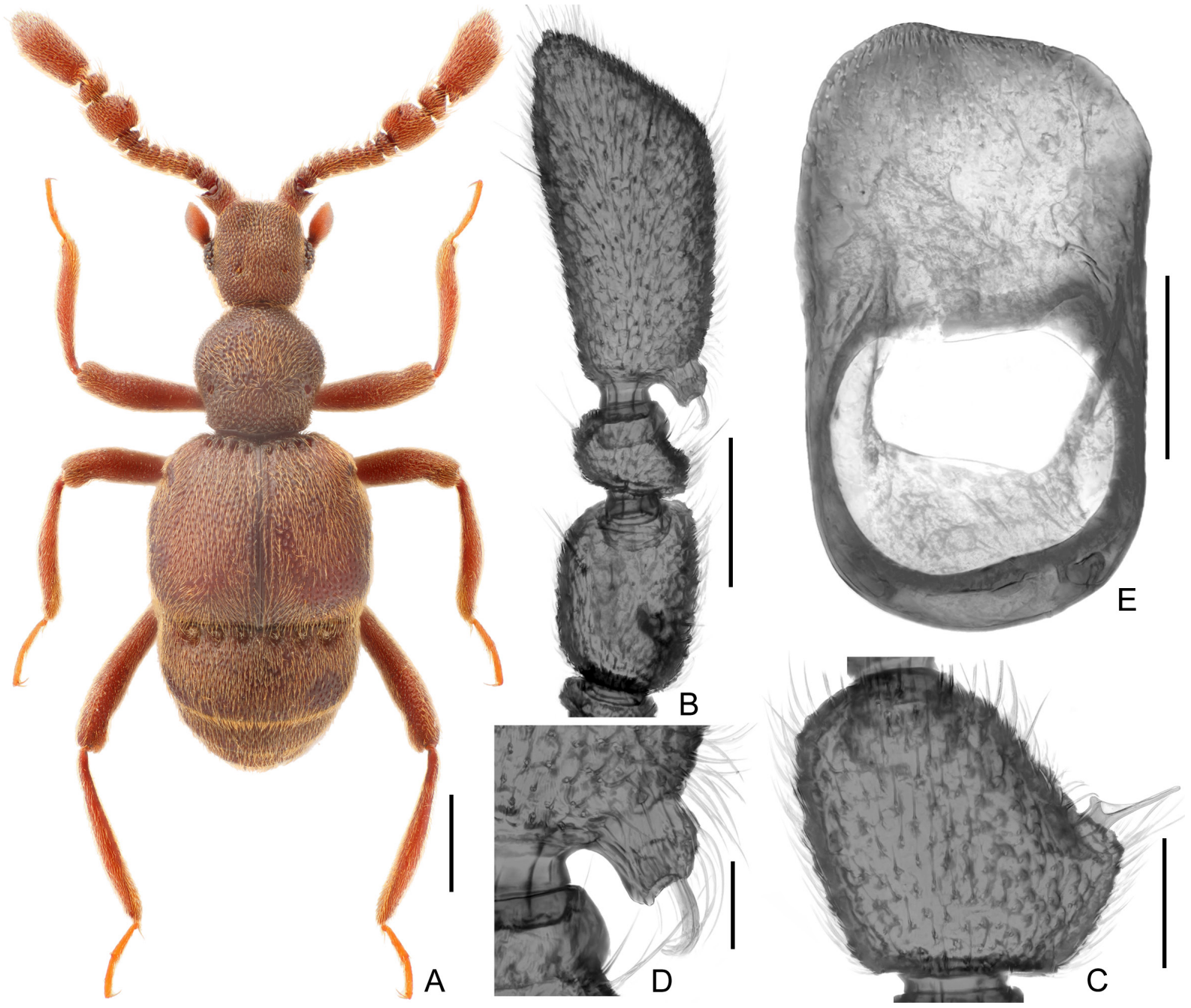

Fig. 3. Sathytes chayuensis sp. nov., $\widehat{\partial}$. A. Dorsal habitus. B. Antennal club. C. Antennomere 9. D. Modification of antennomere 11, enlarged. E. Aedeagus, ventral view. Scale bars: $A=0.5 \mathrm{~mm} ; \mathrm{B}=$ $0.2 \mathrm{~mm} ; \mathrm{C}, \mathrm{E}=0.1 \mathrm{~mm} ; \mathrm{D}=0.05 \mathrm{~mm}$. 
Elytra strongly transverse. Abdomen widest at tergite 1 (IV) and narrowing apically. Aedeagus (Fig. 3E) flattened and slightly asymmetrical.

\section{Female}

MEASUREMENTS. $\mathrm{AnL}=1.42 \mathrm{~mm} ; \mathrm{AL}=0.91 \mathrm{~mm} ; \mathrm{AW}=1.07 \mathrm{~mm} ; \mathrm{TBL}=3.05 \mathrm{~mm} ; \mathrm{EL}=0.98 \mathrm{~mm}$; $\mathrm{EW}=1.16 \mathrm{~mm} ; \mathrm{HL}=0.67 \mathrm{~mm} ; \mathrm{HW}=0.63 \mathrm{~mm} ; \mathrm{LE}=0.19 \mathrm{~mm} ; \mathrm{LT}=0.21 \mathrm{~mm} ; \mathrm{PL}=0.71 \mathrm{~mm} ; \mathrm{PW}=$ $0.71 \mathrm{~mm}$.

Slightly larger than male in size. Each eye composed of about 15 facets; LE:LT $=0.9$. Antennal clubs lacking modifications.

\section{Distribution}

China: Xizang.

$$
\begin{aligned}
& \text { Sathytes chengzhifeii sp. nov. } \\
& \text { urn:1sid:zoobank.org:act:3DA68CF9-8416-4B33-A31B-C583A45AEC52 }
\end{aligned}
$$

Fig. 4

\section{Differential diagnosis}

The male of $S$. chengzhifeii sp. nov. is most similar to that of $S$. huapingensis sp. nov. from Guangxi, China and S. larifuga Shen \& Yin, 2019 from Sabah, Borneo, with which it shares the unique, subtriangular basal protuberance of antennomere 11. The new species can be separated from the former by the larger body size $(2.69-2.81 \mathrm{~mm}$ vs $2.49 \mathrm{~mm})$ and the more basal and more distal positions of the protuberance and apophysis of antennomeres 11 and 9, respectively; and from the latter by the relatively much smaller eyes in relation to the head, more elongate antennomeres 9 and 11, and more distal position of the apophysis of antennomere 9 .

\section{Etymology}

The new species is named after Zhi-Fei Cheng, one of the collectors of the new species.

Type material (5 specimens)

\section{Holotype}

CHINA - 今; Yunnan, Dehongmang (德宏芒市), Yingjiang County (盈江县), Tongbiguan Town (铜壁

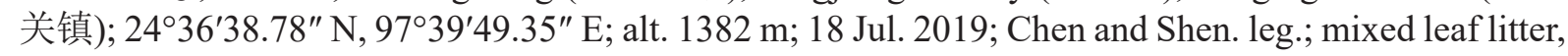
sifted; SNUC.

\section{Paratypes}

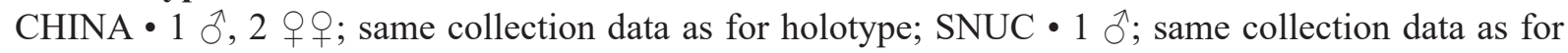
holotype; near Mangna Road (芒那公路); 2436'59.75" N, 9744'30.89" E; alt. 1550 m, 29 Jul. 2019; SNUC.

\section{Description}

\section{Male}

Measurements. AeL $=0.25 \mathrm{~mm} ; \mathrm{AnL}=1.25-1.29 \mathrm{~mm} ; \mathrm{AL}=0.73-0.78 \mathrm{~mm} ; \mathrm{AW}=0.86-0.89 \mathrm{~mm}$; $\mathrm{TBL}=2.69-2.81 \mathrm{~mm} ; \mathrm{EL}=0.81-0.84 \mathrm{~mm} ; \mathrm{EW}=0.98-1.02 \mathrm{~mm} ; \mathrm{HL}=0.57-0.59 \mathrm{~mm} ; \mathrm{HW}=0.55-$ $0.57 \mathrm{~mm} ; \mathrm{LE}=0.21 \mathrm{~mm} ; \mathrm{LT}=0.11 \mathrm{~mm} ; \mathrm{PL}=0.58-0.60 \mathrm{~mm} ; \mathrm{PW}=0.57-0.58 \mathrm{~mm}$.

Body (Fig. 4A) reddish-brown, mouthparts and tarsi paler. Head approximately as long as wide. Each eye composed of about 20 facets; $L E: L T=1.9$. Antennal clubs (Fig. 4B) formed by antennomeres 9-11; antennomere 9 (Fig. 4C) slightly elongate, each with long semi-membranous apophysis at basal $1 / 3$ of 
mesal margin; antennomere 10 strongly transverse, obconical; antennomere 11 (Fig. 4B) about 1.8 times as long as wide, each with single subtriangular and blunt basal protuberance covered with short tuft of setae at apex (Fig. 4D). Pronotum about as long as wide. Elytra strongly transverse. Abdomen widest at tergite 1 (IV) and narrowing apically. Aedeagus (Fig. 4E) flattened, slightly elongate, and slightly asymmetrical at apex.

\section{Female}

Measurements. $\mathrm{AnL}=1.27 \mathrm{~mm} ; \mathrm{AL}=0.86 \mathrm{~mm} ; \mathrm{AW}=0.95 \mathrm{~mm} ; \mathrm{TBL}=3.0 \mathrm{~mm} ; \mathrm{EL}=0.83 \mathrm{~mm}$; $\mathrm{EW}=1.05 \mathrm{~mm} ; \mathrm{HL}=0.62 \mathrm{~mm} ; \mathrm{HW}=0.60 \mathrm{~mm} ; \mathrm{LE}=0.17 \mathrm{~mm} ; \mathrm{LT}=0.16 \mathrm{~mm} ; \mathrm{PL}=0.69 \mathrm{~mm} ; \mathrm{PW}=$ $0.65 \mathrm{~mm}$.

Similar to male in size. Each eye composed of about 14 facets; LE: LT = 1.6. Antennal clubs lacking modifications.

\section{Distribution}

China: Yunnan.

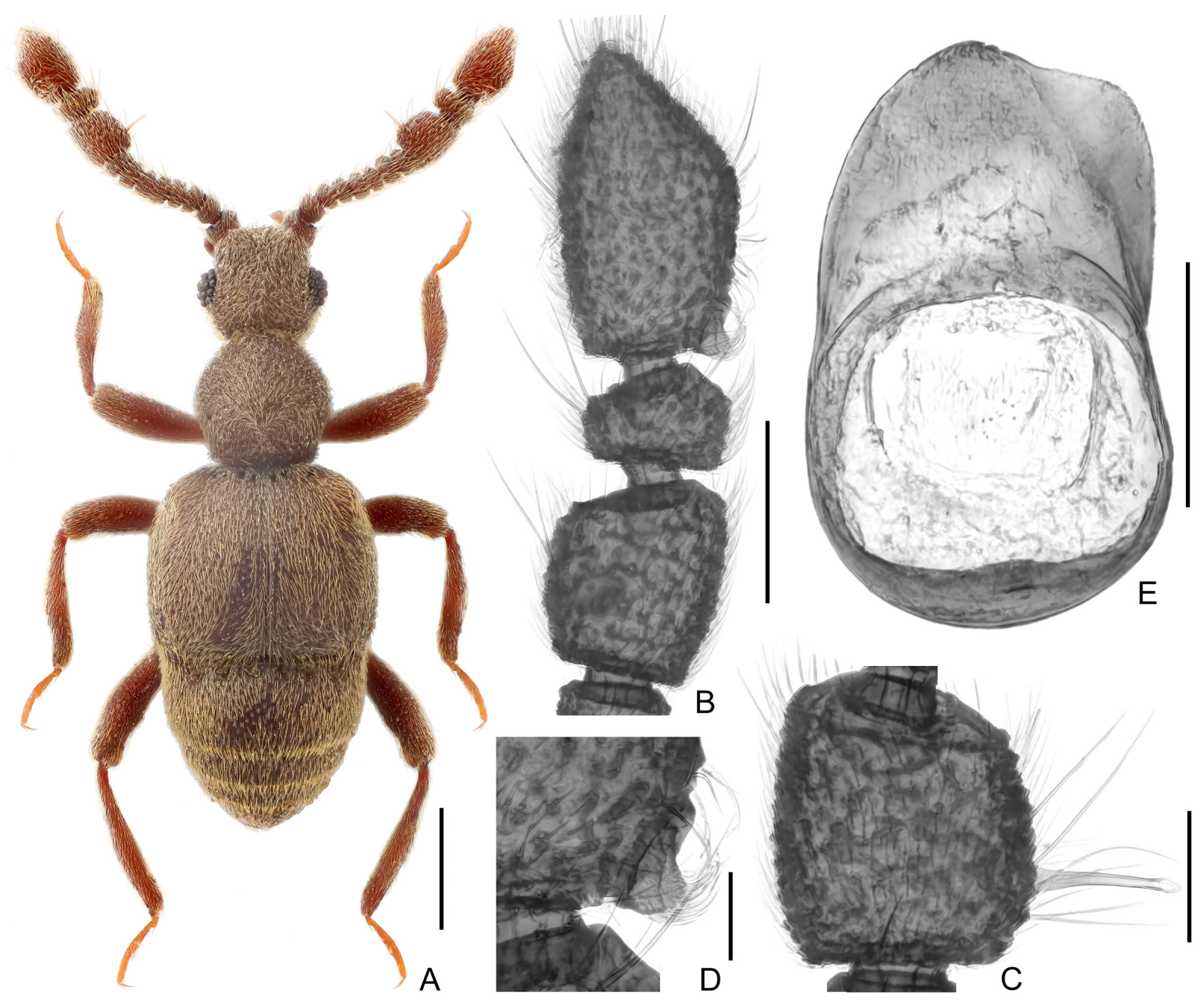

Fig. 4. Sathytes chengzhifeii sp. nov., Ô. A. Dorsal habitus. B. Antennal club. C. Antennomere 9. D. Modification of antennomere 11, enlarged. E. Aedeagus, ventral view. Scale bars: $A=0.5 \mathrm{~mm}$; $=$ $0.2 \mathrm{~mm} ; \mathrm{C}, \mathrm{E}=0.1 \mathrm{~mm}$; $=0.05 \mathrm{~mm}$. 
Sathytes huapingensis sp. nov.

urn:lsid:zoobank.org:act:BD4DFA36-F5F8-452C-8CB6-7D8276F97154

Fig. 5

\section{Differential diagnosis}

The male of $S$. huapingensis sp. nov. possesses a roundly triangular basal protuberance of antennomere 11, similar to that of S. chengzhifeii sp. nov. (China: Yunnan) and S. larifuga (Borneo: Sabah). Sathytes huapingensis sp. nov. can be separated from S. chengzhifeii sp. nov. by the smaller body size (2.49 $\mathrm{mm}$ vs $2.69-2.81 \mathrm{~mm}$ ), and the more distal and more basal positions of the protuberance and apophysis of antennomeres 11 and 9, respectively; and from S. larifuga by the relatively much smaller eyes in relation to the head, much narrower apical portion of the median lobe of the aedeagus, and a different distribution.

\section{Etymology}

The new species is named after Huaping Nature Reserve, where the type locality of this species is situated.

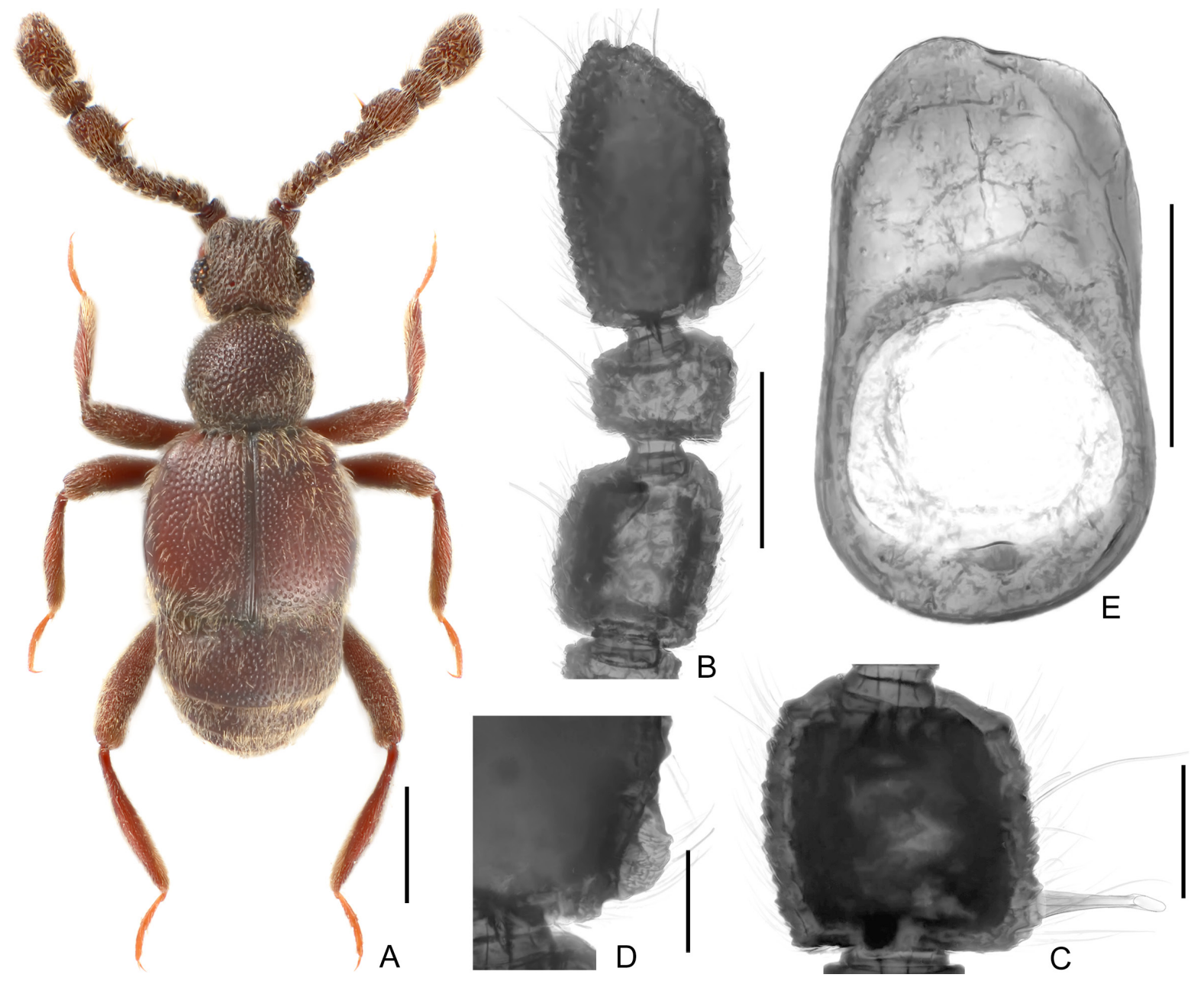

Fig. 5. Sathytes huapingensis sp. nov., O. A. Dorsal habitus. B. Antennal club. C. Antennomere 9. D. Modification of antennomere 11, enlarged. E. Aedeagus, ventral view. Scale bars: $\mathrm{A}=0.5 \mathrm{~mm} ; \mathrm{B}=$ $0.2 \mathrm{~mm} ; \mathrm{C}, \mathrm{E}=0.1 \mathrm{~mm} ; \mathrm{D}=0.05 \mathrm{~mm}$. 
Type material (1 specimen)

Holotype

CHINA • 今 ; Guangxi Prov, Lingui County (临桂县), Huaping N.R. (花坪自然保护区), Anjiangping (安江坪); alt. 1200 m; 13-16 Jul. 2011; Tang L. and He W.-J. leg.; SNUC.

\section{Description}

Male

Measurements. AeL $=0.25 \mathrm{~mm} ; \mathrm{AnL}=1.39 \mathrm{~mm} ; \mathrm{AL}=0.61 \mathrm{~mm} ; \mathrm{AW}=0.80 \mathrm{~mm}$;BL $=2.49 \mathrm{~mm}$; $\mathrm{EL}=0.82 \mathrm{~mm} ; \mathrm{EW}=0.93 \mathrm{~mm} ; \mathrm{HL}=0.48 \mathrm{~mm} ; \mathrm{HW}=0.53 \mathrm{~mm} ; \mathrm{LE}=0.18 \mathrm{~mm} ; \mathrm{LT}=0.13 \mathrm{~mm} ; \mathrm{PL}=$ $0.58 \mathrm{~mm} ; \mathrm{PW}=0.55 \mathrm{~mm}$.

Body (Fig. 5A) reddish-brown, mouthparts and tarsi paler. Head slightly wider than long. Each eye composed of about 16 facets; LE: LT = 1.4. Antennal clubs (Fig. 5B) formed by antennomeres 9-11; antennomere 9 (Fig. 5C) sub-quadrate, each with semi-membranous apophysis at basal 1/5 of mesal margin; antennomere 10 strongly transverse, obconical; antennomere 11 (Fig. 5B) about 1.5 times as long as wide, each with single large, blunt, short protuberance close to bases, covered with sparse setae at apex (Fig. 5D). Pronotum slightly longer than broad. Elytra strongly transverse. Abdomen widest at tergite 1 (IV) and narrowing apically. Aedeagus (Fig. 5E) elongate and slightly asymmetrical at apex.

\section{Female}

Unknown.

\section{Distribution}

China: Guangxi.

$$
\begin{gathered}
\text { Sathytes linzhiensis sp. nov. } \\
\text { urn:1sid:zoobank.org:act:5B7777C6-80EB-4E6A-B54E-A4255CE82C81 }
\end{gathered}
$$

Fig. 6

\section{Differential diagnosis}

The male of this new species shares with S. perpusillus Yin \& Li, 2012, S. vafelus Löbl, 1979 and S. minutus Löbl, 1979 the antennomere 11 bearing two small protuberances at the base of the mesal margin. This new species has the largest body size $(1.70-1.91 \mathrm{~mm})$, and can be readily separated from S. perpusillus $(\mathrm{TBL}=1.48-1.52 \mathrm{~mm}$ ) by the more distal position of the apophysis on antennomere 9 ; from $S$. vafelus (TBL 1.4-1.5 mm) by the relatively shorter antennomere 11 (length/width $=1.6$ ) and less protruding basal protuberance on it; and from S. minutus $(\mathrm{TBL}=1.3 \mathrm{~mm})$ by the much larger body size and more relatively elongate antennomere 11 .

\section{Etymology}

The new species is named after Linzhi City, where the type locality of this species is situated.

Type material (4 specimens)

\section{Holotype}

CHINA - O; Xizang, Motuo County, path from Bari village (巴日村) to Renqingbeng Temple (仁青崩寺); 29¹8'44.82" N, 95²1'27.18” E; alt. 1833 m; 19 Mar. 2017; X.-B. Song leg.; SNUC.

\section{Paratypes}

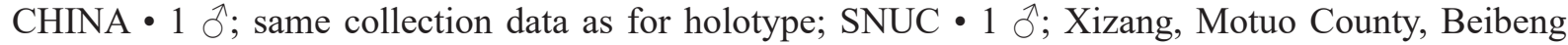
Village (背崩乡); 30¹4'52" N, 9447'55" E; alt. 1700-1900 m; 5 Aug. 2014; Wen-Xuan Bi leg.; SNUC

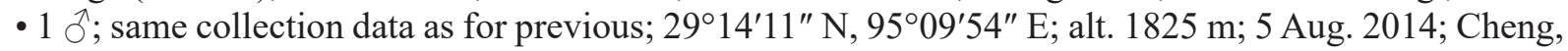
Peng and Shen leg.; mixed leaf litter, sifted; SNUC. 


\section{Description}

\section{Male}

Measurements. AeL $=0.16 \mathrm{~mm} ; \mathrm{AnL}=0.79-0.82 \mathrm{~mm} ; \mathrm{AL}=0.47-0.55 \mathrm{~mm} ; \mathrm{AW}=0.52-0.57 \mathrm{~mm}$; $\mathrm{TBL}=1.70-1.91 \mathrm{~mm} ; \mathrm{EL}=0.47-0.55 \mathrm{~mm} ; \mathrm{EW}=0.61-0.65 \mathrm{~mm} ; \mathrm{HL}=0.37-0.42 \mathrm{~mm} ; \mathrm{HW}=0.36-$ $0.41 \mathrm{~mm} ; \mathrm{LE}=0.15 \mathrm{~mm} ; \mathrm{LT}=0.09 \mathrm{~mm} ; \mathrm{PL}=0.39-0.41 \mathrm{~mm} ; \mathrm{PW}=0.38-0.40 \mathrm{~mm}$.

Body (Fig. 6A) reddish-brown, mouthparts and tarsi paler. Head about as long as wide. Each eye composed of about 22 facets; LE: LT = 1.7. Antennal clubs (Fig. 6B) formed by antennomeres 9-11; antennomere 9 (Fig. 6C) each with mesal margin shorter than lateral margin, long semi-membranous apophysis at middle of mesal surface; antennomere 10 strongly transverse, obconical; antennomere 11 (Fig. 6B) about 1.6 times as long as wide, each with two small basal protuberances covered with long apical setae (Fig. 6D). Pronotum about as long as wide. Elytra strongly transverse. Abdomen widest at tergite 1 (IV) and narrowing apically. Aedeagus (Fig. 6E) almost symmetrical, with slightly angulate apex.
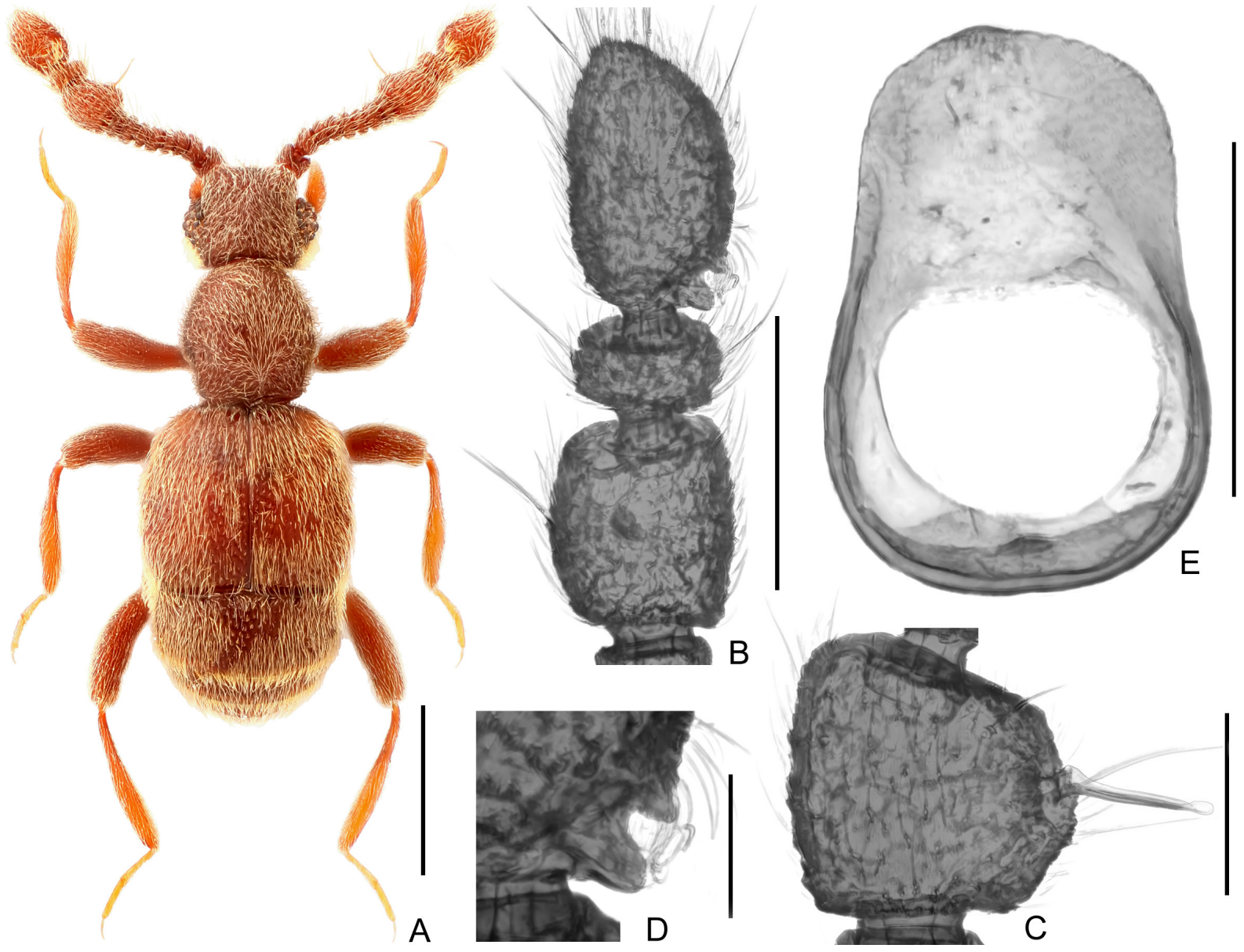

Fig. 6. Sathytes linzhiensis sp. nov., ठ̊ (population of Beibeng). A. Dorsal habitus. B. Antennal club. C. Antennomere 9. D. Modification of antennomere 11, enlarged. E. Aedeagus, ventral view. Scale bars: $\mathrm{A}=0.5 \mathrm{~mm} ; \mathrm{B}=0.2 \mathrm{~mm} ; \mathrm{C}, \mathrm{E}=0.1 \mathrm{~mm} ; \mathrm{D}=0.05 \mathrm{~mm}$. 
Female

Unknown.

\section{Distribution}

China: Xizang.

Sathytes longitrabis Yin \& Li, 2012

Fig. 7

Sathytes longitrabis Yin \& Li in Yin et al. 2012: 843, figs 1a, 3d, 4d, 7d, 8d.

Material examined (8 specimens)

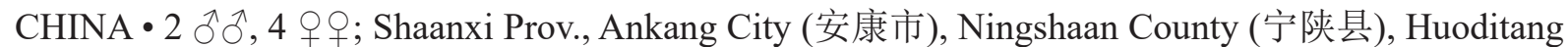
Forestry Centre (火地塘林场); 33 $26^{\prime} \mathrm{N}, 108^{\circ} 27^{\prime} \mathrm{E}$; alt. 1500-1700 m; 12 Jul. 2012; Chen and W.R. Li leg.; SNUC • 1 Ō, 1 q, same collection data as for previous; $33^{\circ} 26^{\prime} 02^{\prime \prime} \mathrm{N}, 108^{\circ} 27^{\prime} 07^{\prime \prime} \mathrm{E}$; alt. 1500-1700 m; 12 Jul. 2012; Y.H. Pan leg.; mixed leaf litter, sifted; SNUC.
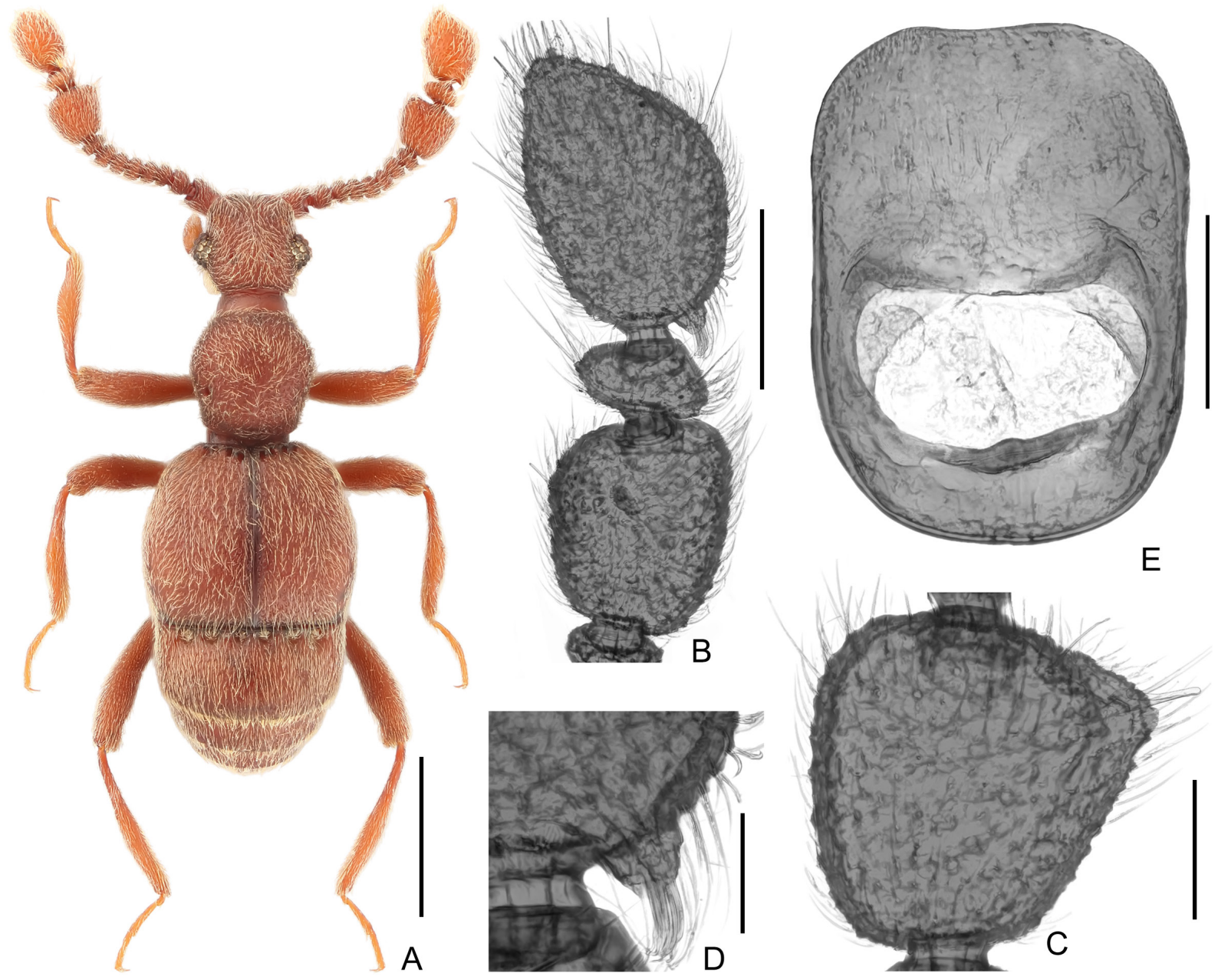

E
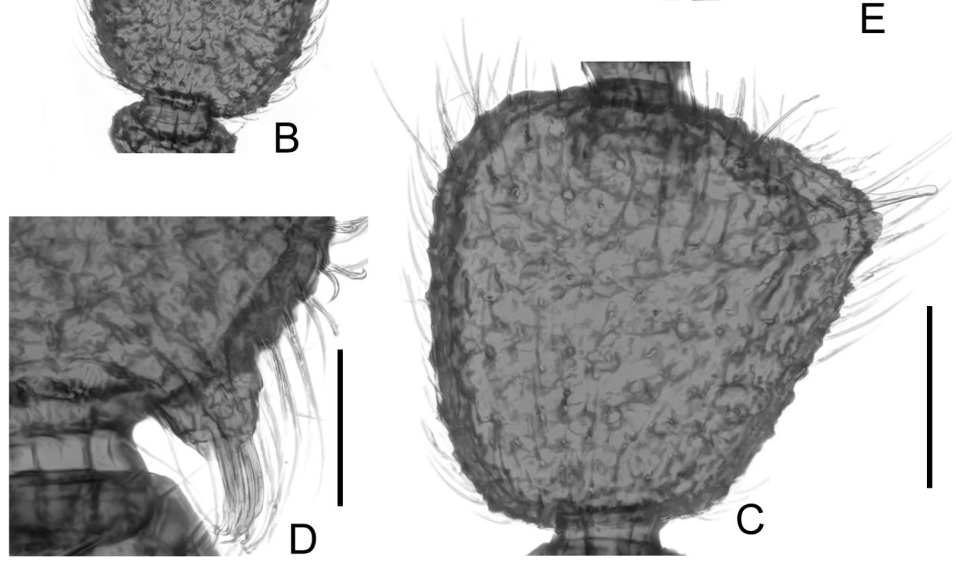

Fig. 7. Sathytes longitrabis, ð̊ (population of Huoditang). A. Dorsal habitus. B. Antennal club. C. Antennomere 9. D. Modification of antennomere 11, enlarged. E. Aedeagus, ventral view. Scale bars: $\mathrm{A}=0.5 \mathrm{~mm} ; \mathrm{B}=0.2 \mathrm{~mm} ; \mathrm{C}, \mathrm{E}=0.1 \mathrm{~mm} ; \mathrm{D}=0.05 \mathrm{~mm}$. 


\section{Remarks}

This species was originally described based on a single male specimen from Houzhenzi, Qinling (秦岭), and is here recorded from Huoditang in the same mountain range. The habitus and diagnostic characters are shown in Fig. 7.

\section{Distribution}

China: Shaanxi.

\section{Sathytes maoershanus sp. nov.}

urn:1sid:zoobank.org:act:6BDDF95B-909A-466C-BD0E-95F78C7FE400

Fig. 8

\section{Differential diagnosis}

The male of the new species shares with S. larinus Yin \& Li, 2012 from Zhejiang and Fujian a similar form of the antennal modification, but can be clearly separated by the slightly more transverse pronotum and much larger body size (2.96-3.07 vs $2.17 \mathrm{~mm})$.

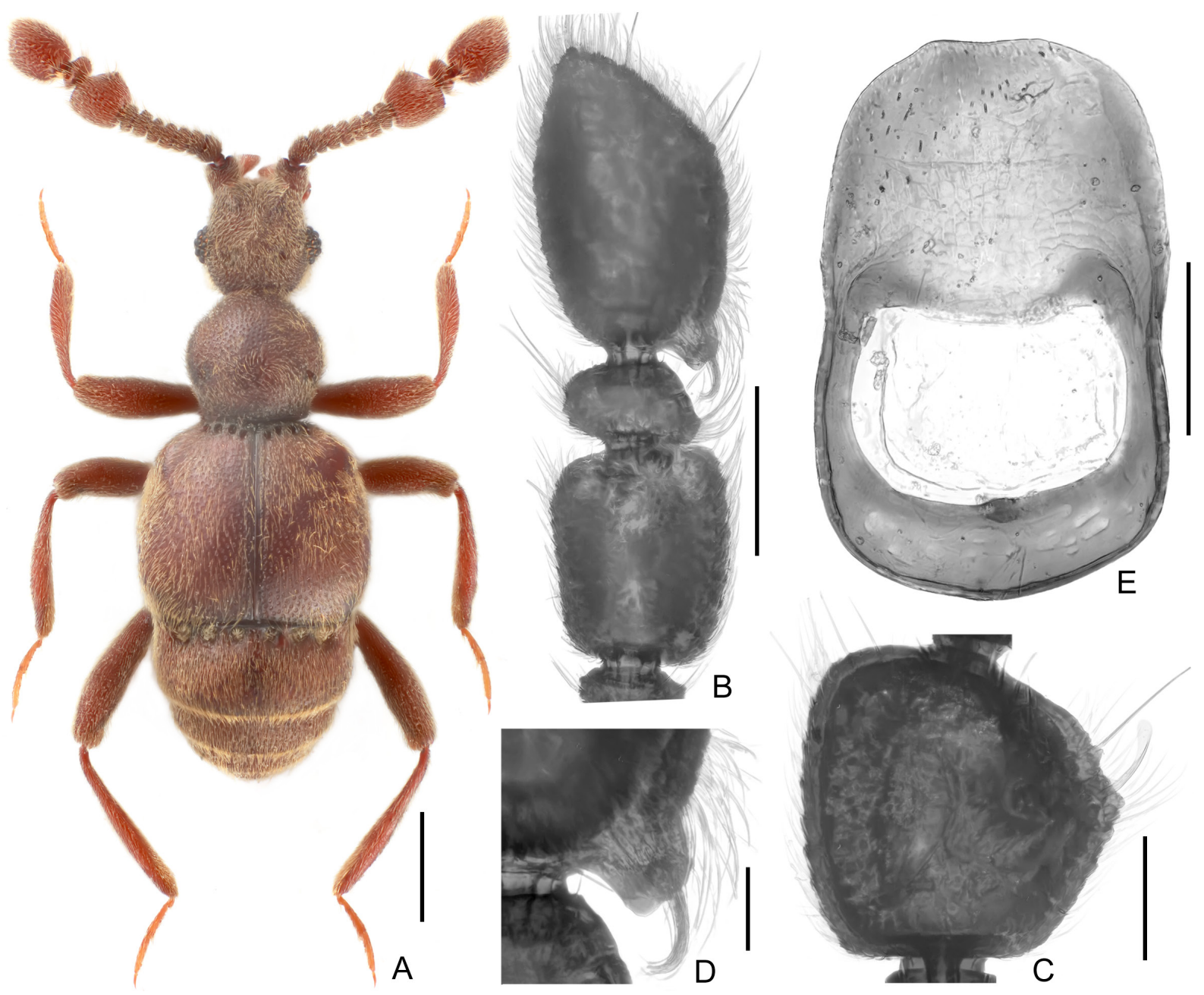

Fig. 8. Sathytes maoershanus sp. nov., Ô. A. Dorsal habitus. B. Antennal club. C. Antennomere 9. D. Modification of antennomere 11, enlarged. E. Aedeagus, ventral view. Scale bars: $\mathrm{A}=0.5 \mathrm{~mm}$; $=$ $0.2 \mathrm{~mm} ; \mathrm{C}, \mathrm{E}=0.1 \mathrm{~mm} ; \mathrm{D}=0.05 \mathrm{~mm}$. 


\section{Etymology}

The new species is named after Maoershan Nature Reserve, where the type locality of this species is situated.

Type material (27 specimens)

\section{Holotype}

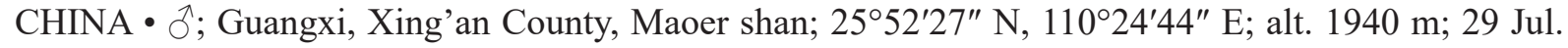
2014; Peng, Song, Yu and Yan leg.; beech forest, mixed leaf litter, humus, sifted; SNUC.

\section{Paratypes}

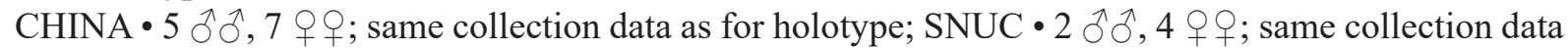
as for holotype; $25^{\circ} 52^{\prime} 31^{\prime \prime} \mathrm{N}, 110^{\circ} 24^{\prime} 54^{\prime \prime} \mathrm{E}$; alt. 1950-2000 m; 23 Jul. 2012; Hu and Song leg.; mixed

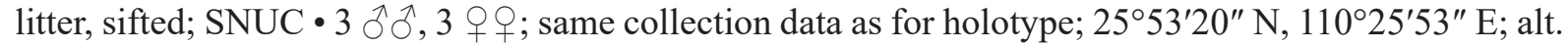
2000-2100 m; 22 Jul. 2012; Hu and Song leg.; mixed litter, sifted; SNUC • 2 우; same collection data as for holotype; $25^{\circ} 54^{\prime} 52^{\prime \prime} \mathrm{N}, 110^{\circ} 27^{\prime} 14^{\prime \prime} \mathrm{E}$; alt. 1550-1750 m; 24 Jul. 2012; Hu and Song leg.; mixed litter, sifted; SNUC.

\section{Description}

\section{Male}

Measurements. AeL $=0.32 \mathrm{~mm} ; \mathrm{AnL}=1.39-1.52 \mathrm{~mm} ; \mathrm{AL}=0.77-0.81 \mathrm{~mm} ; \mathrm{AW}=0.90-0.95 \mathrm{~mm}$; $\mathrm{TBL}=2.96-3.07 \mathrm{~mm} ; \mathrm{EL}=0.95-0.98 \mathrm{~mm} ; \mathrm{EW}=1.09-1.11 \mathrm{~mm} ; \mathrm{HL}=0.61-0.62 \mathrm{~mm} ; \mathrm{HW}=0.59$ $0.60 \mathrm{~mm} ; \mathrm{LE}=0.18 \mathrm{~mm} ; \mathrm{LT}=0.19 \mathrm{~mm} ; \mathrm{PL}=0.63-0.66 \mathrm{~mm} ; \mathrm{PW}=0.60-0.62 \mathrm{~mm}$.

Body (Fig. 8A) reddish-brown, mouthparts and tarsi paler. Head approximately as long as wide. Each eye composed of about 20 facets; LE: LT $=0.9$. Antennal clubs (Fig. 8B) formed by antennomeres 9-11; antennomere 9 (Fig. 8C) each with semi-membranous apophysis at middle of mesal surface; antennomere 10 strongly transverse, obconical; antennomere 11 (Fig. 8B) about 1.7 times as long as wide, each with large apically truncate protuberance bearing tuft of long apical setae at base (Fig. 8D). Pronotum slightly longer than broad. Elytra transverse. Abdomen widest at tergite 1 (IV) and narrowing apically. Aedeagus (Fig. 8E) slightly constricted near middle, almost symmetrical.

\section{Female}

Measurements. AnL $=1.26-1.29 \mathrm{~mm} ; \mathrm{AL}=0.86-0.94 \mathrm{~mm} ; \mathrm{AW}=1.05-1.08 \mathrm{~mm} ; \mathrm{TBL}=3.01-3.14 \mathrm{~mm}$; $\mathrm{EL}=0.87-0.89 \mathrm{~mm} ; \mathrm{EW}=1.11-1.12 \mathrm{~mm} ; \mathrm{HL}=0.61-0.63 \mathrm{~mm} ; \mathrm{HW}=0.59-0.60 \mathrm{~mm} ; \mathrm{LE}=0.17 \mathrm{~mm}$; $\mathrm{LT}=0.19 \mathrm{~mm} ; \mathrm{PL}=0.67-0.68 \mathrm{~mm} ; \mathrm{PW}=0.64-0.66 \mathrm{~mm}$.

Similar to male in size. Each eye composed of about 12 facets; LE: LT =0.9. Antennal clubs lacking modifications.

\section{Distribution}

China: Guangxi.

Sathytes nujiangensis sp. nov. urn:1sid:zoobank.org:act:2E5BC920-834F-42DC-9FB4-99550406183F

Fig. 9

\section{Differential diagnosis}

The male of S. nujiangensis sp. nov. is morphologically similar to that of S. transversus sp. nov. (China: Xizang), S. valentulus sp. nov. (China: Guangxi) and S. usitatus Yin \& Li, 2012 (China: Yunnan) in the 
presence of a single basal protuberance of antennomere 11, and the markedly transverse antennomere 9 (when widths are fully visible). It can be separated from S. transversus sp. nov. by the much smaller body size ( $2.17 \mathrm{~mm}$ vs $2.61-2.72 \mathrm{~mm}$ ) and less protuberant mesal margin of antennomere 9; from S. valentulus sp. nov. by the thinner antennomere 11 each with a relatively longer basal protuberance, and more transverse antennomere 9; and from S. usitatus by the more oblique anterior margin of antennomere 9 and the more medial positions of the apophyses on these antennomeres.

\section{Etymology}

The new species is named after $\mathrm{Nu}$ Jiang, where the type locality of this species is situated.

Type material (1 specimen)

\section{Holotype}

CHINA - 万; Yunnan, Gongshan Dulong Nu Autonomous Co. (贡山独龙族怒族自治县), Qiutan Vil. (邱滩村), Langwang Temple (朗汪寺); 2804'24.60" N, 98³4'45.73" E; alt. 1510 m; 28 Jul. 2015; Wen-Xuan Bi leg.; SNUC.

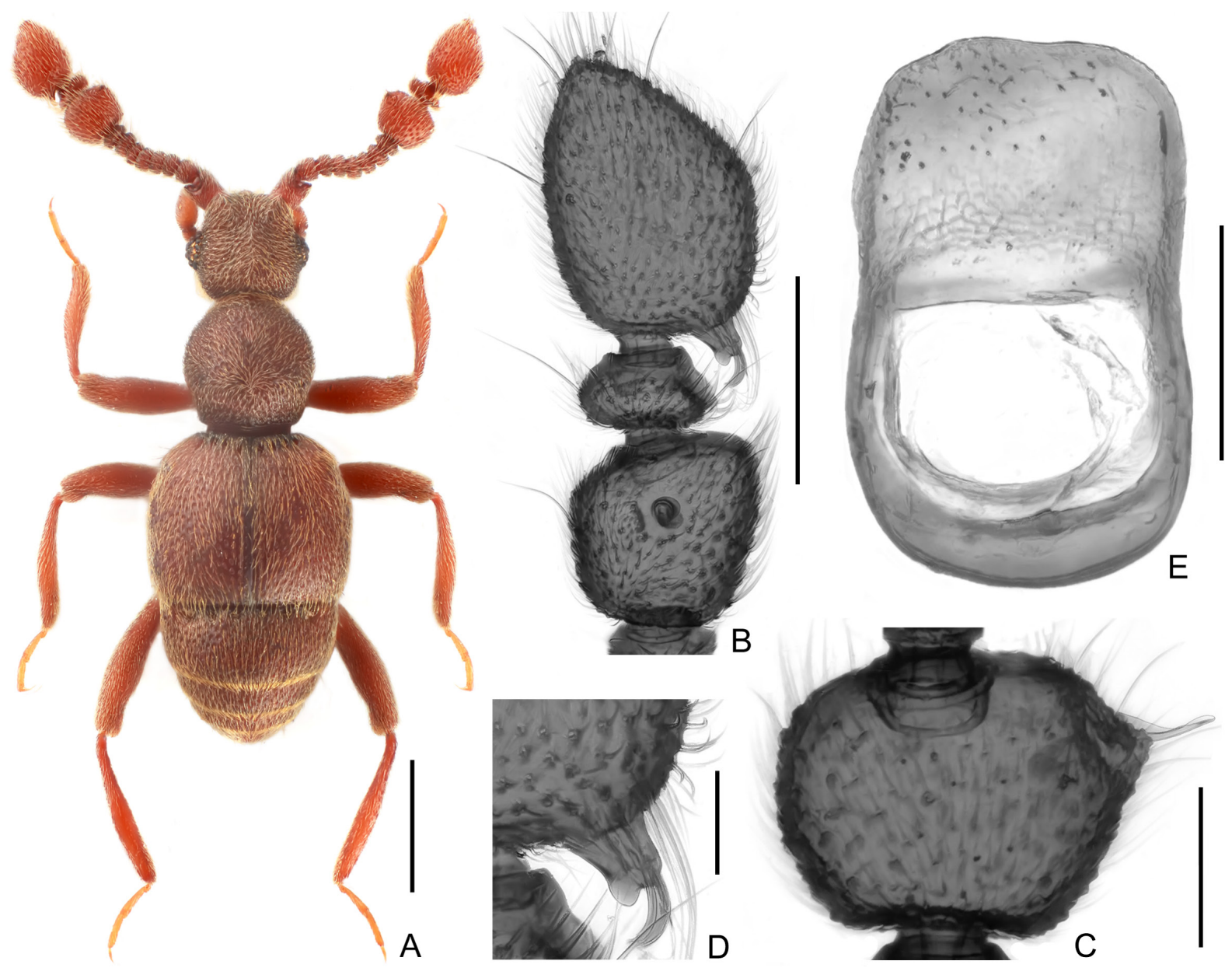

Fig. 9. Sathytes nujiangensis sp. nov., §ิ. A. Dorsal habitus. B. Antennal club. C. Antennomere 9. D. Modification of antennomere 11, enlarged. E. Aedeagus, ventral view. Scale bars: $A=0.5 \mathrm{~mm} ; \mathrm{B}=$ $0.2 \mathrm{~mm} ; \mathrm{C}, \mathrm{E}=0.1 \mathrm{~mm} ; \mathrm{D}=0.05 \mathrm{~mm}$. 


\section{Description}

\section{Male}

Measurements. AeL $=0.24 \mathrm{~mm} ; \mathrm{AnL}=1.12 \mathrm{~mm} ; \mathrm{AL}=0.53 \mathrm{~mm} ; \mathrm{AW}=0.67 \mathrm{~mm} ; \mathrm{TBL}=2.17 \mathrm{~mm}$; $\mathrm{EL}=0.65 \mathrm{~mm} ; \mathrm{EW}=0.77 \mathrm{~mm} ; \mathrm{HL}=0.48 \mathrm{~mm} ; \mathrm{HW}=0.46 \mathrm{~mm} ; \mathrm{LE}=0.16 \mathrm{~mm} ; \mathrm{LT}=0.13 \mathrm{~mm} ; \mathrm{PL}=$ $0.51 \mathrm{~mm} ; \mathrm{PW}=0.49 \mathrm{~mm}$.

Body (Fig. 9A) reddish-brown, mouthparts and tarsi paler. Head slightly longer than wide. Each eye composed of about 20 facets; LE: LT = 1.2. Antennal clubs (Fig. 9B) formed by antennomeres 9-11; antennomere 9 (Fig. 9C) strongly transverse, broadest at apical $1 / 3$, each with semi-membranous apophysis at apex of expansion, and at approximately apical $1 / 3$ of mesal margin; antennomere 10 strongly transverse, obconical; antennomere 11 (Fig. 9B) about 1.5 times as long as wide, each with single apically truncate basal protuberance bearing tuft of long setae at the apex. (Fig. 9D). Pronotum slightly longer than broad. Elytra moderately transverse. Abdomen widest at tergite 1 (IV) and narrowing apically. Aedeagus (Fig. 9E) slightly elongate, slightly constricted at middle and almost symmetrical.

\section{Female}

Unknown.

\section{Distribution}

China: Yunnan.

Sathytes panzhaohuii sp. nov. urn:lsid:zoobank.org:act:F8372711-164B-4B3F-B9EC-98B98BA84813

Fig. 10

\section{Differential diagnosis}

The male of $S$. panzhaohuii sp. nov. has antennomere 11 each bearing a wide basal protuberance with two tufts of apical setae, a condition unique within the genus which allows ready identification of the new species.

\section{Etymology}

The new species is named after Prof Zhao-Hui Pan, who kindly provided logistic support during our field work in Xizang.

\section{Type material (17 specimens)}

\section{Holotype}

CHINA • ^’; Xizang, Nyingchi (林芝市), Pailong Country (排龙乡); 300'34.71" N, 9457'57.64" E; alt. 2190 m; 15 Jul.-1 Aug. 2019; Z.-W. Yin leg.; FIT (flight intercept trap); SNUC.

\section{Paratypes}

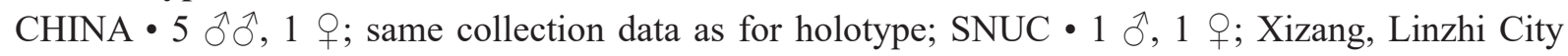
(林芝市), near Changxi Vill. (昌西村); 30¹4'52" N, 94²7'55" E; alt. 2380 m; 7 Jul. 2018; Cheng, Peng and Shen leg.; mixed leaf litter, sifted; SNUC • 3 우; same collection data as for previous; near Pailong

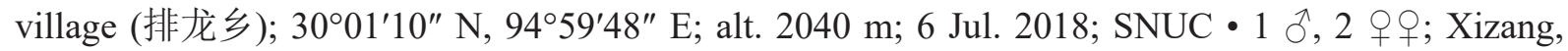

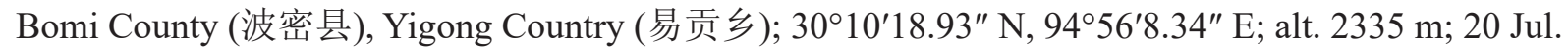
2019; Z.-W. Yin leg.; leaf litter / fungus; SNUC・ 2 ふへ ; Xizang, Chayu Co., Xiachayu To., Talin Bridge; 28.53340539 N, $96.97800188^{\circ}$ E; alt. 1541 m; 30 Jul. 2019; Liang Lü leg.; SNUC. 


\section{Description}

\section{Male}

Measurements. AeL $=0.19 \mathrm{~mm} ; \mathrm{AnL}=1.05-1.07 \mathrm{~mm} ; \mathrm{AL}=0.54-0.56 \mathrm{~mm} ; \mathrm{AW}=0.72-0.85 \mathrm{~mm}$; $\mathrm{TBL}=2.13-2.24 \mathrm{~mm} ; \mathrm{EL}=0.63-0.65 \mathrm{~mm} ; \mathrm{EW}=0.82-0.85 \mathrm{~mm} ; \mathrm{HL}=0.48-0.51 \mathrm{~mm} ; \mathrm{HW}=0.44$ $0.46 \mathrm{~mm} ; \mathrm{LE}=0.15 \mathrm{~mm} ; \mathrm{LT}=0.17 \mathrm{~mm} ; \mathrm{PL}=0.48-0.52 \mathrm{~mm} ; \mathrm{PW}=0.46-0.49 \mathrm{~mm}$.

Body (Fig. 10A) reddish-brown, mouthparts and tarsi paler. Head moderately longer than wide. Each eye composed of about 18 facets; LE:LT $=0.9$. Antennal clubs (Fig. 10B) formed by antennomeres 9-11; antennomere 9 (Fig. 10C) moderately elongate, each with long semi-membranous apophysis at apical $2 / 5$ of mesal margin; antennomere 10 strongly transverse, obconical; antennomere 11 (Fig. 10B) about 1.6 times as long as wide, each with single broad basal protuberance covered with two tufts of setae at apex (Fig. 10D). Pronotum about as long as broad. Elytra transverse. Abdomen widest at tergite 1 (IV) and narrowing apically. Aedeagus (Fig. 10E) slightly broad and almost symmetrical.

\section{Female}

Measurements. $\mathrm{AnL}=0.94-1.04 \mathrm{~mm} ; \mathrm{AL}=0.54-0.56 \mathrm{~mm} ; \mathrm{AW}=0.72-0.85 \mathrm{~mm} ; \mathrm{TBL}=2.12-2.37 \mathrm{~mm}$; $\mathrm{EL}=0.58-0.68 \mathrm{~mm} ; \mathrm{EW}=0.81-0.90 \mathrm{~mm} ; \mathrm{HL}=0.52-0.58 \mathrm{~mm} ; \mathrm{HW}=0.46-0.50 \mathrm{~mm} ; \mathrm{LE}=0.13 \mathrm{~mm}$; $\mathrm{LT}=0.21 \mathrm{~mm} ; \mathrm{PL}=0.48-0.55 \mathrm{~mm} ; \mathrm{PW}=0.46-0.53 \mathrm{~mm}$.
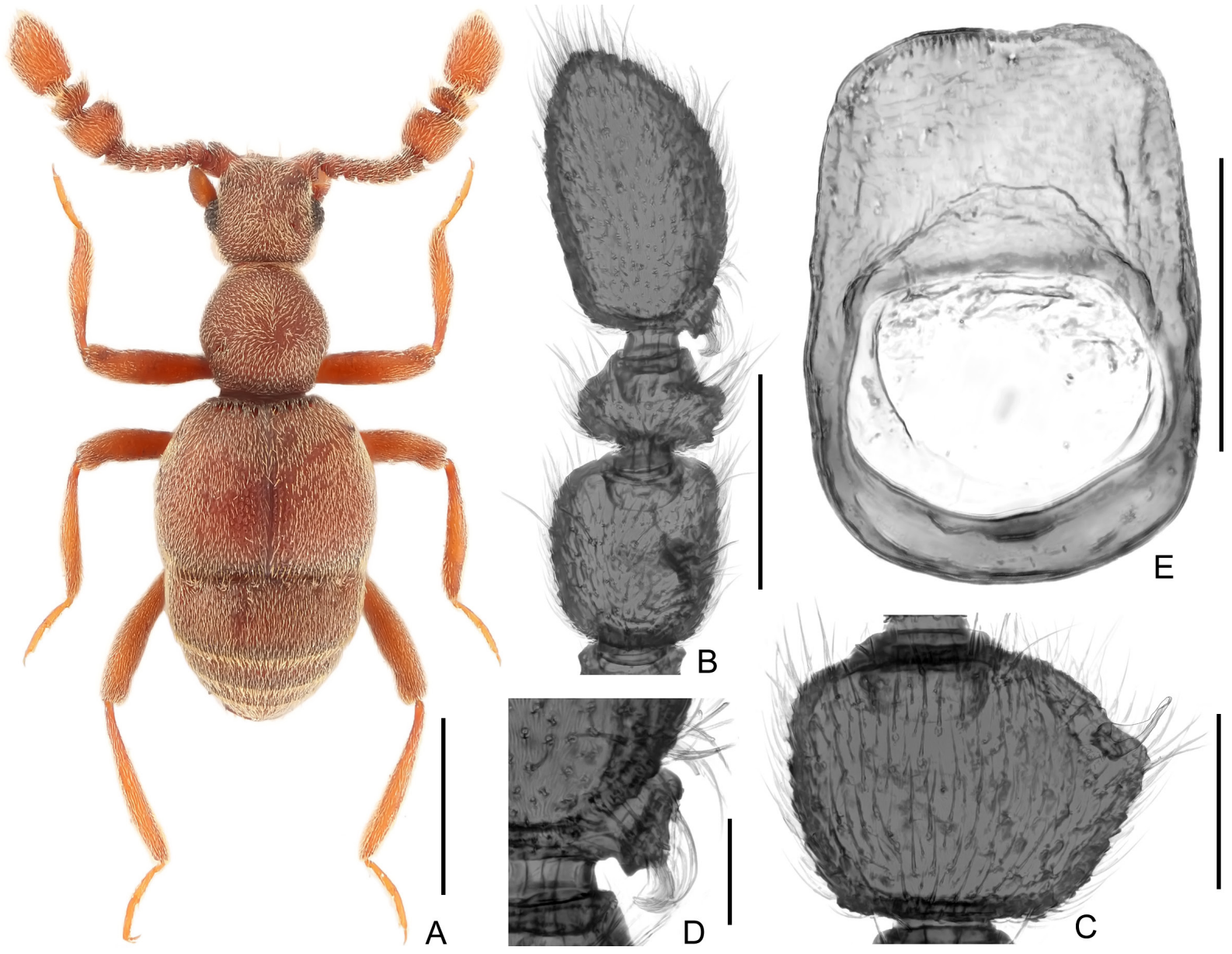

Fig. 10. Sathytes panzhaohuii sp. nov., §̊ (population of Pailong). A. Dorsal habitus. B. Antennal club. C. Antennomere 9. D. Modification of antennomere 11, enlarged. E. Aedeagus, ventral view. Scale bars: $\mathrm{A}=0.5 \mathrm{~mm} ; \mathrm{B}=0.2 \mathrm{~mm} ; \mathrm{C}, \mathrm{E}=0.1 \mathrm{~mm} ; \mathrm{D}=0.05 \mathrm{~mm}$. 
Similar to male in size. Each eye composed of about 14 facets; LE: LT $=0.6$. Antennal clubs lacking modifications.

\section{Distribution}

China: Xizang.

Sathytes shennong sp. nov. urn:lsid:zoobank.org:act:A4C78BE1-C7DE-41DF-9CBE-46B851946BE7

Fig. 11

\section{Differential diagnosis}

Sathytes shennong sp. nov. shares with $S$. chayuensis sp. nov., S. xingdoumontis sp. nov., S. magnus, $S$. tangliangi and $S$. grandis the single basal protuberance of antennomere 11 and the conspicuously oblique mesal margin of antennomere 9. It differs from S. chayuensis sp. nov. by the much less elongate antennomere 11; from $S$. xingdoumontis sp. nov. by the more protuberant mesal margin of antennomere 9 ; and from the latter three species by the much smaller body size $(2.29 \mathrm{~mm}$ vs $3.36 \mathrm{~mm}$ of $S$. magnus, $2.8 \mathrm{~mm}$ of $S$. tangliangi and $3 \mathrm{~mm}$ of $S$. grandis) and a similar but different form of antennomere 9 .

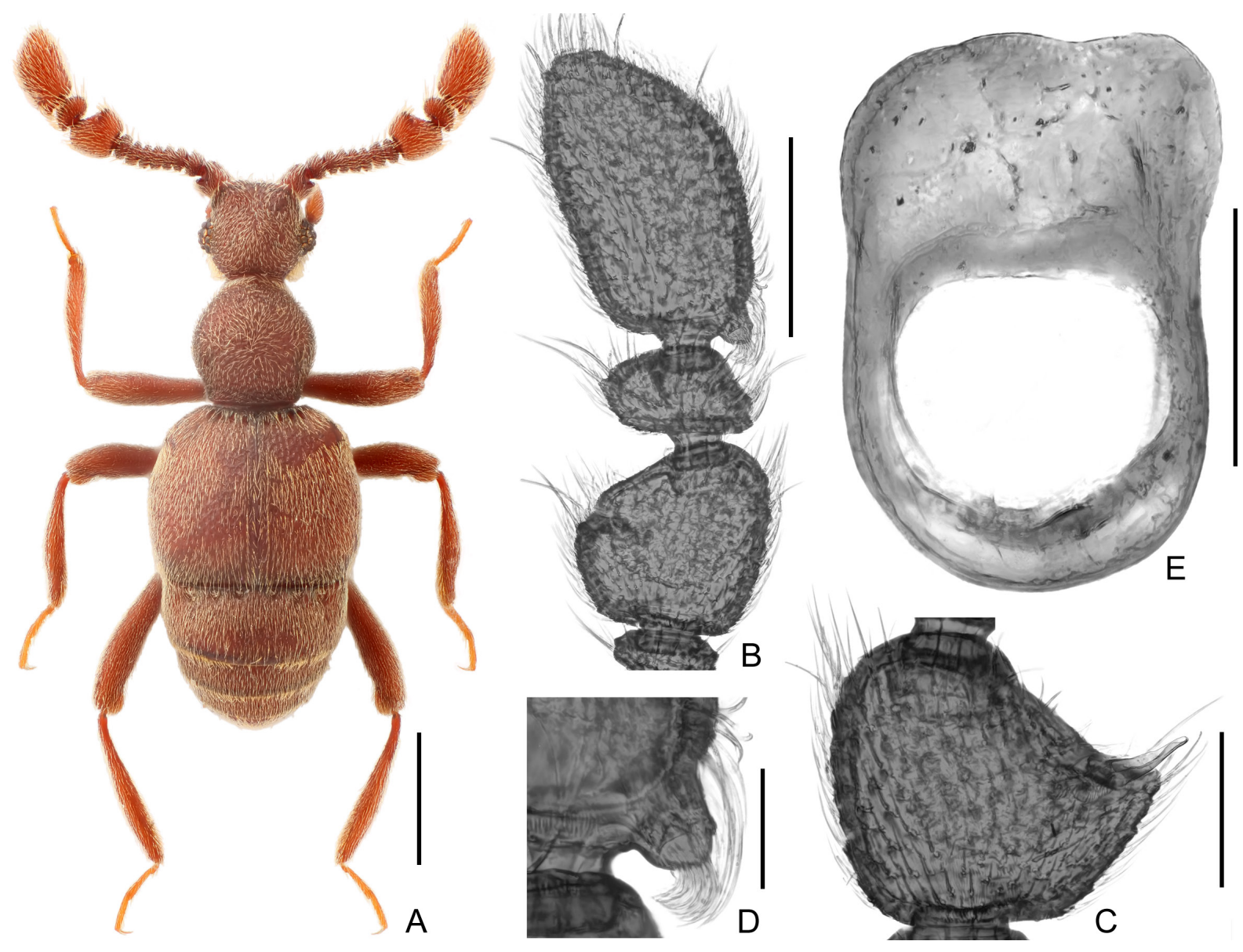

Fig. 11. Sathytes shennong sp. nov., วิ. A. Dorsal habitus. B. Antennal club. C. Antennomere 9. D. Modification of antennomere 11, enlarged. E. Aedeagus, ventral view. Scale bars: $A=0.5 \mathrm{~mm} ; \mathrm{B}=$ $0.2 \mathrm{~mm} ; \mathrm{C}, \mathrm{E}=0.1 \mathrm{~mm} ; \mathrm{D}=0.05 \mathrm{~mm}$. 


\section{Etymology}

The new specific epithet 'shennong' (神农) refers to the mythological Chinese deity, from which the name of Shennongjia Nature Reserve originates.

Type material (4 specimens)

\section{Holotype}

CHINA - O; Hubei, Badong Co. (巴东县), Yanduhe To. (沿渡河镇), Xiaoshennongjia Vil. (小神农 架村); $31.3406^{\circ} \mathrm{N}, 110.4131^{\circ}$ E; alt. 1320 m; 27 Apr. 2016; Zhou and Hong-Bin Liang leg.; beating; IZCAS.

\section{Paratypes}

CHINA -3 + $\odot$; same collection data as for holotype; IZCAS.

\section{Description}

\section{Male}

Measurements. $\mathrm{AeL}=0.21 \mathrm{~mm} ; \mathrm{AnL}=1.05 \mathrm{~mm} ; \mathrm{AL}=0.60 \mathrm{~mm} ; \mathrm{AW}=0.72 \mathrm{~mm} ; \mathrm{TBL}=2.29 \mathrm{~mm}$; $\mathrm{EL}=0.68 \mathrm{~mm} ; \mathrm{EW}=0.84 \mathrm{~mm} ; \mathrm{HL}=0.50 \mathrm{~mm} ; \mathrm{HW}=0.47 \mathrm{~mm} ; \mathrm{LE}=0.16 \mathrm{~mm} ; \mathrm{LT}=0.17 \mathrm{~mm} ; \mathrm{PL}=$ $0.51 \mathrm{~mm} ; \mathrm{PW}=0.50 \mathrm{~mm}$.

Body (Fig. 11A) reddish-brown, mouthparts and tarsi paler. Head slightly longer than broad. Each eye composed of about 22 facets; $L E: L T=0.9$. Antennal clubs (Fig. 11B) formed by antennomeres 9-11; mesal margin of antennomere 9 (Fig. 11C) oblique and angularly expanded, with semi-membranous apophysis anterior to apex of expansion; antennomere 10 strongly transverse, obconical; antennomere 11 (Fig. 11B) about 1.7 times as long as wide, each with single short basal protuberance covered with tuft of setae at apex (Fig. 11D). Pronotum about as long as wide. Elytra moderately transverse. Abdomen widest at tergite 1 (IV) and narrowing apically. Aedeagus (Fig. 11E) slightly asymmetrical, broadened at apex.

\section{Female}

Measurements. AnL $=0.95-0.98 \mathrm{~mm} ; \mathrm{AL}=0.79-0.82 \mathrm{~mm} ; \mathrm{AW}=0.76-0.79 \mathrm{~mm} ; \mathrm{TBL}=2.42-2.49 \mathrm{~mm}$; $\mathrm{EL}=0.63-0.64 \mathrm{~mm} ; \mathrm{EW}=0.83-0.85 \mathrm{~mm} ; \mathrm{HL}=0.49-0.51 \mathrm{~mm} ; \mathrm{HW}=0.46-0.48 \mathrm{~mm} ; \mathrm{LE}=0.15 \mathrm{~mm}$; $\mathrm{LT}=0.18 \mathrm{~mm} ; \mathrm{PL}=0.51-0.52 \mathrm{~mm} ; \mathrm{PW}=0.50-0.51 \mathrm{~mm}$.

Slightly larger than male in size. Each eye composed of about 14 facets; LE: LT $=0.8$. Antennal clubs lacking modifications.

\section{Distribution}

China: Hubei.

Sathytes tangliangi Yin \& Li, 2012

Sathytes yunnanicus Yin \& Li in Yin et al. 2012: 850, figs 2b, 3k, 5e, 7k, 8k.

Material examined (1 specimen)

CHINA 1 1 ; Yunnan, Baoshan City, Tengchong County, Mingguang Town, Baihua Ling; $25^{\circ} 18^{\prime} 10^{\prime \prime} \mathrm{N}$, 9847'31" E; alt. 1870 m; 21 Apr. 2013; Dai, Song and Peng leg.; SNUC. 


\section{Remarks}

This species was originally described based on a male and a female from Qiqi, Gongshan County. An additional specimen shows it has a wider distribution in Yunnan.

\section{Distribution}

China: Yunnan.

Sathytes tianquanus sp. nov.

urn:1sid:zoobank.org:act:3303B110-9BE0-49BE-B87D-6E22C868629F

Fig. 12

\section{Differential diagnosis}

The male of $S$. tianquanus sp. nov. is morphologically close to that of $S$. obliquus Shen \& Yin, 2020 (Zhejiang) and S. longitrabis (Shaanxi) in the apophysis of antennomere 9 located at anterior half of the segment. It differs from S. obliquus by the much more transverse antennomere 9; and from S. longitrabis

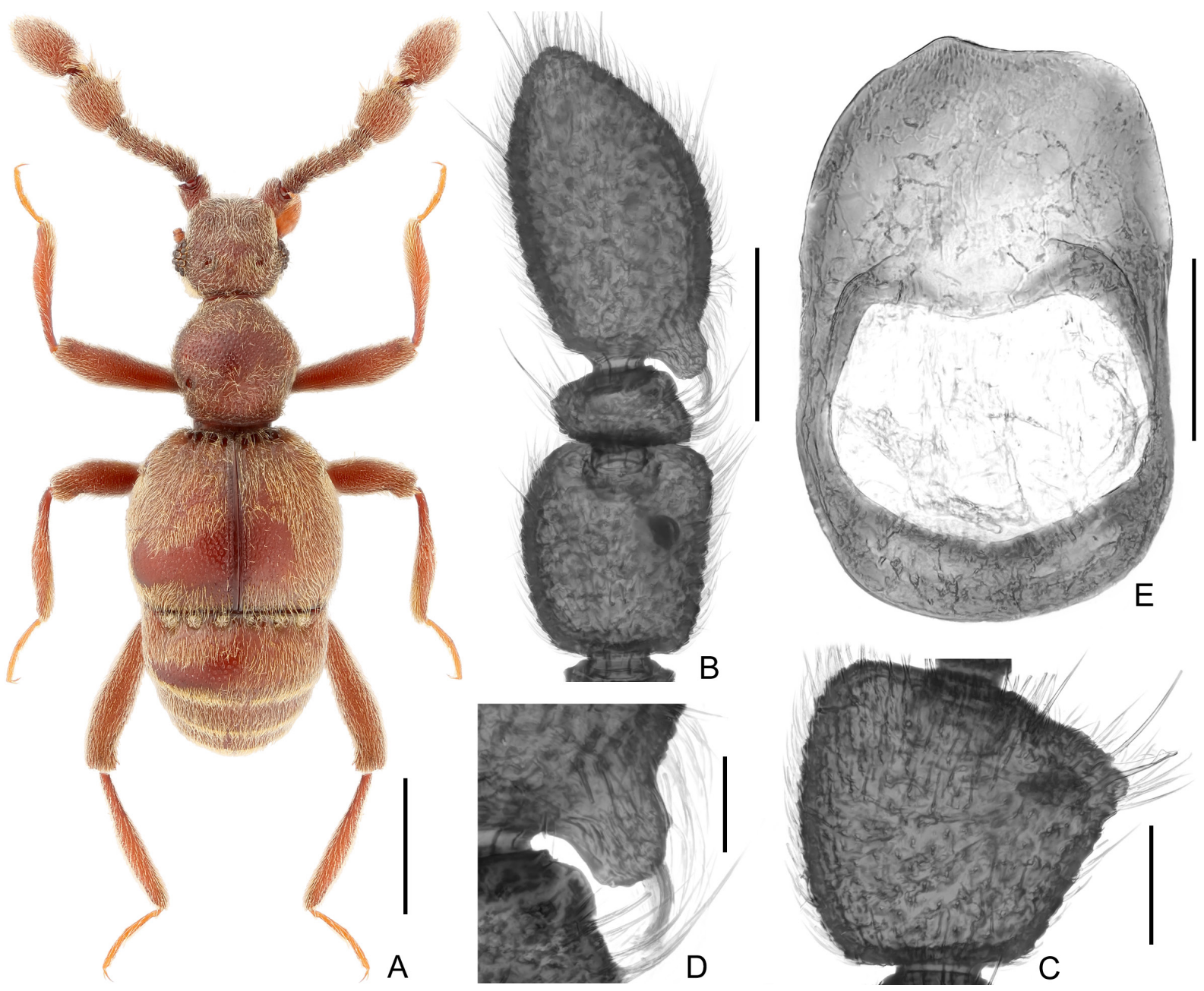

Fig. 12. Sathytes tianquanus sp. nov., Ô. A. Dorsal habitus. B. Antennal club. C. Antennomere 9. D. Modification of antennomere 11, enlarged. E. Aedeagus, ventral view. Scale bars: A $=0.5 \mathrm{~mm} ; \mathrm{B}=$ $0.2 \mathrm{~mm} ; \mathrm{C}, \mathrm{E}=0.1 \mathrm{~mm} ; \mathrm{D}=0.05 \mathrm{~mm}$. 
by the larger body size (2.92 $\mathrm{mm}$ vs $2.21-2.42 \mathrm{~mm})$, more elongate antennomere 11 and more transverse antennomere 9 .

\section{Etymology}

The new species is named after Tianquan County, where the type locality of this species is situated.

Type material ( 5 specimens)

\section{Holotype}

CHINA - O’; Sichuan, Tianquan (天全县), Er'lang Shan (二郎山); 2952'23" N, 10248'34" E; alt. 2200-2300 m; 13 Jul. 2012; Z. Peng and Z.-W. Yin leg.; old pass; leaf litter, sifted; SNUC.

\section{Paratypes}

CHINA $\bullet 4$ 9 ; same collection data as for holotype; SNUC.

\section{Description}

\section{Male}

Measurements. AeL $=0.32 \mathrm{~mm} ; \mathrm{AnL}=1.34 \mathrm{~mm} ; \mathrm{AL}=0.79 \mathrm{~mm} ; \mathrm{AW}=0.92 \mathrm{~mm}$;BL $=2.92 \mathrm{~mm}$; $\mathrm{EL}=0.89 \mathrm{~mm} ; \mathrm{EW}=1.07 \mathrm{~mm} ; \mathrm{HL}=0.61 \mathrm{~mm} ; \mathrm{HW}=0.58 \mathrm{~mm} ; \mathrm{LE}=0.19 \mathrm{~mm} ; \mathrm{LT}=0.18 \mathrm{~mm} ; \mathrm{PL}=$ $0.63 \mathrm{~mm} ; \mathrm{PW}=0.63 \mathrm{~mm}$.

Body (Fig. 12A) reddish-brown, mouthparts and tarsi paler. Head slightly longer than wide. Each eye composed of about 22 facets; LE: $\mathrm{LT}=1.1$. Antennal clubs (Fig. 12B) formed by antennomeres 9-11; antennomere 9 (Fig. 12C) broadest at apical $1 / 3$, each with semi-membranous apophysis at apex of mesal expansion; antennomere 10 strongly transverse, obconical; antennomere 11 (Fig. 12B) about 1.6 times as long as wide, each with large basal protuberance bearing tuft of long apical setae at apex (Fig. 12D). Pronotum about as long as wide. Elytra moderately transverse. Abdomen widest at tergite 1 (IV) and narrowing apically. Aedeagus (Fig. 12E) slightly asymmetrical at apex.

\section{Female}

Measurements. AnL $=1.20-1.21 \mathrm{~mm} ; \mathrm{AL}=0.90-0.91 \mathrm{~mm} ; \mathrm{AW}=0.89-0.90 \mathrm{~mm} ; \mathrm{TBL}=2.81-2.85 \mathrm{~mm}$; $\mathrm{EL}=0.74-0.75 \mathrm{~mm} ; \mathrm{EW}=0.96-0.97 \mathrm{~mm} ; \mathrm{HL}=0.57-0.58 \mathrm{~mm} ; \mathrm{HW}=0.53-0.54 \mathrm{~mm} ; \mathrm{LE}=0.15 \mathrm{~mm}$; $\mathrm{LT}=0.16 \mathrm{~mm} ; \mathrm{PL}=0.60-0.61 \mathrm{~mm} ; \mathrm{PW}=0.60-0.61 \mathrm{~mm}$.

Slightly smaller than male in size. Each eye composed of about 16 facets; LE: LT $=0.9$. Antennal clubs lacking modifications.

\section{Distribution}

China: Sichuan.

Sathytes transversus sp. nov. urn:1sid:zoobank.org:act:92D74AE8-1C41-46C5-BFD6-0AE8F334E6FB

Fig. 13

\section{Differential diagnosis}

The male of S. transversus sp. nov. is most similar to that of S. usitatus from Yunnan in the similar form of the antennomeres 9 and 11, but can be clearly separated form it by the larger body size $(2.61-2.72 \mathrm{~mm}$ vs $2.23-2.42 \mathrm{~mm}$ ), more posteriorly located apophysis of antennomere 9 and longer basal protuberance of antennomere 11 .

\section{Etymology}

The specific name refers to the strongly transverse antennomere 9 of the male. 
Type material (7 specimens)

\section{Holotype}

CHINA • O'; Xizang, Nyingchi, Pailong Country; 300'34.71" N, 9457'57.64" E; alt. 2190 m; 15 Jul.1 Aug. 2019, Z.-W. Yin leg.; FIT (flight intercept trap); SNUC.

\section{Paratypes}

CHINA • $3 \widehat{\partial} \widehat{\partial}, 1$ क; same collection data as for holotype; SNUC $\bullet 1$ \%; Xizang, Linzhi City, near Pailong village; $30^{\circ} 01^{\prime} 10^{\prime \prime}$ N, 94 59'48" E; alt. 2040 m; 6 Jul. 2018; Cheng, Peng and Shen leg.; mixed leaf litter, sifted; SNUC - 1 ơ; Xizang, Motuo County, near $80 \mathrm{~K} ; 29^{\circ} 41^{\prime} 09^{\prime \prime} \mathrm{N}, 95^{\circ} 30^{\prime} 10^{\prime \prime} \mathrm{E}$; alt. 2330 m; 9 Jul. 2018; Cheng, Peng and Shen leg.; mixed leaf litter, sifted; SNUC.

\section{Description}

\section{Male}

Measurements. AeL $=0.24 \mathrm{~mm} ; \mathrm{AnL}=1.19-1.22 \mathrm{~mm} ; \mathrm{AL}=0.73-0.75 \mathrm{~mm} ; \mathrm{AW}=0.82-0.83 \mathrm{~mm}$; $\mathrm{TBL}=2.61-2.72 \mathrm{~mm} ; \mathrm{EL}=0.74-0.77 \mathrm{~mm} ; \mathrm{EW}=0.96-0.99 \mathrm{~mm} ; \mathrm{HL}=0.55-0.58 \mathrm{~mm} ; \mathrm{HW}=0.51-$ $0.53 \mathrm{~mm} ; \mathrm{LE}=0.17 \mathrm{~mm} ; \mathrm{LT}=0.16 \mathrm{~mm} ; \mathrm{PL}=0.59-0.62 \mathrm{~mm} ; \mathrm{PW}=0.53-0.57 \mathrm{~mm}$.

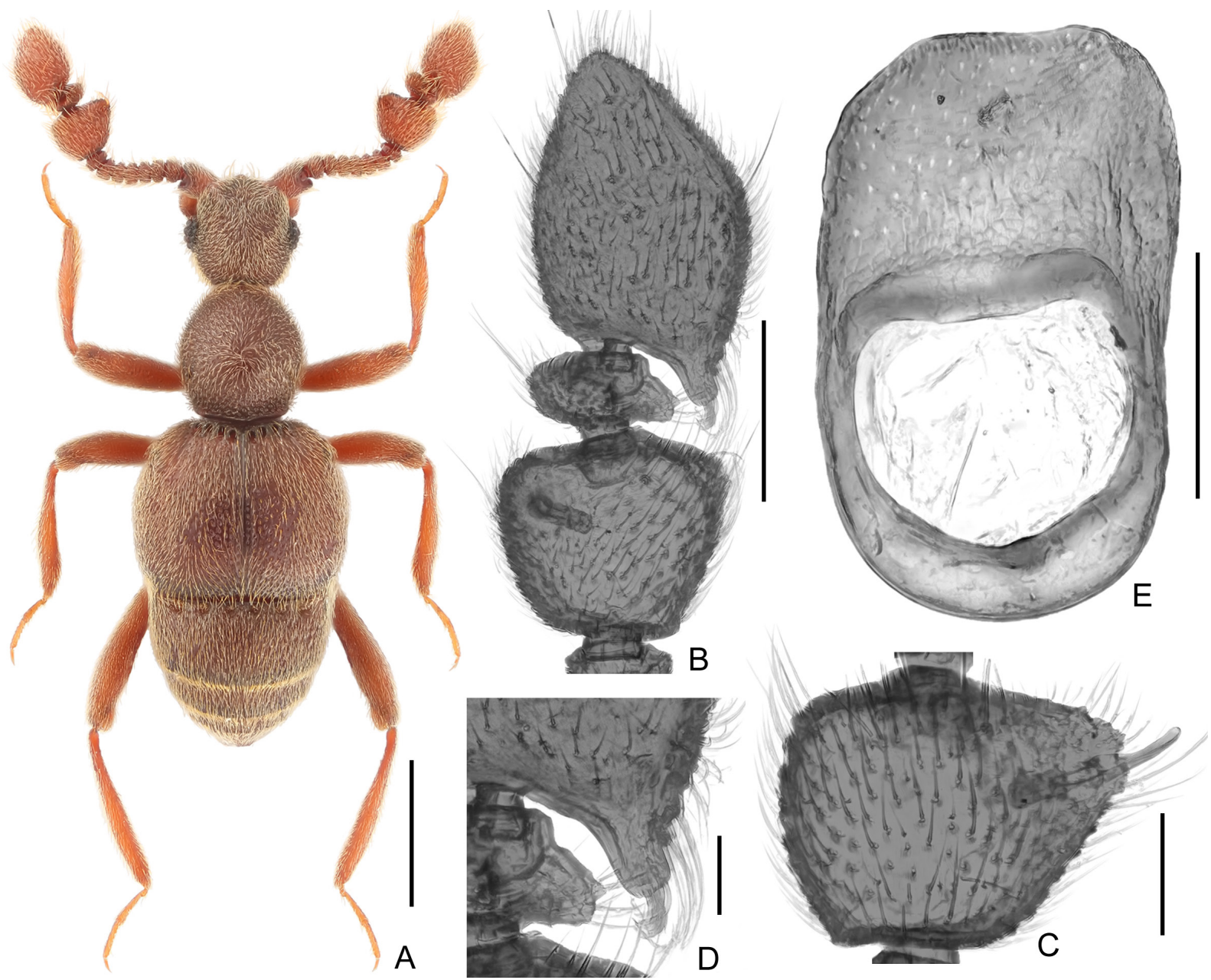

Fig. 13. Sathytes transversus sp. nov., $\widehat{\partial}$ (population from Pailong). A. Dorsal habitus. B. Antennal club. C. Antennomere 9. D. Modification of antennomere 11, enlarged. E. Aedeagus, ventral view. Scale bars: $\mathrm{A}=0.5 \mathrm{~mm} ; \mathrm{B}=0.2 \mathrm{~mm} ; \mathrm{C}, \mathrm{E}=0.1 \mathrm{~mm} ; \mathrm{D}=0.05 \mathrm{~mm}$. 
Body (Fig. 13A) reddish-brown, mouthparts and tarsi paler. Head slightly longer than wide. Each eye composed of about 20 facets; LE:LT = 1.1. Antennal clubs (Fig. 13B) formed by antennomeres 9-11; antennomere 9 (Fig. 13C) strongly transverse, each with semi-membranous apophysis at apex of mesal expansion, and at approximately apical $2 / 5$ of antennomere; antennomere 10 strongly transverse, obconical; antennomere 11 (Fig. 13B) about 1.4 times as long as wide, each with single moderately long basal protuberance covered with tuft of setae at apex (Fig. 13D). Pronotum about slightly longer than wide. Elytra moderately transverse. Abdomen widest at tergite 1 (IV) and narrowing apically. Aedeagus (Fig. 13E) slightly asymmetrical at apex.

\section{Female}

MeAsurements. AnL $=1.16 \mathrm{~mm} ; \mathrm{AL}=0.71-0.72 \mathrm{~mm} ; \mathrm{AW}=0.86-0.87 \mathrm{~mm} ; \mathrm{TBL}=2.52-2.55 \mathrm{~mm}$; $\mathrm{EL}=0.69-0.70 \mathrm{~mm} ; \mathrm{EW}=0.91-0.92 \mathrm{~mm} ; \mathrm{HL}=0.56 \mathrm{~mm} ; \mathrm{HW}=0.49 \mathrm{~mm} ; \mathrm{LE}=0.13 \mathrm{~mm} ; \mathrm{LT}=$ $0.16 \mathrm{~mm} ; \mathrm{PL}=0.56-0.57 \mathrm{~mm} ; \mathrm{PW}=0.55-0.56 \mathrm{~mm}$.

Slightly smaller than male in size. Each eye composed of about 16 facets; LE: LT $=0.8$. Antennal clubs lacking modifications.

\section{Distribution}

China: Xizang.

Sathytes valentulus sp. nov.

urn:1sid:zoobank.org:act:394D438A-130F-4160-B63C-A2E07142B155

Fig. 14

\section{Differential diagnosis}

The male of $S$. valentulus sp. nov. can be readily separated from all congeners by the markedly stout antennomere 11 alone, aided by the unique form of antennomere 9, and its distribution in Guangxi.

\section{Etymology}

The specific name refers to the stout male antennomere 11 of the new species.

Type material (3 specimens)

\section{Holotype}

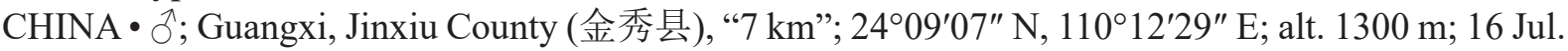
2014; Peng, Song, Yu and Yan leg.; beech forest; mixed leaf litter, humus, sifted; SNUC.

\section{Paratypes}

CHINA $\bullet 1$ के, same collection data as for holotype; SNUC $\bullet 1$; ; same collection data as for holotype;

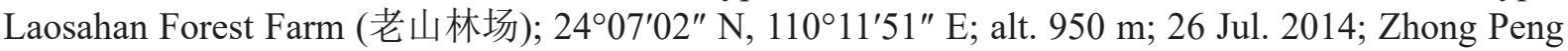
leg.; beech forest, rotten wood, colony of ant; SNUC.

\section{Description}

\section{Male}

MeAsurements. AeL $=0.23 \mathrm{~mm} ; \mathrm{AnL}=1.14-1.17 \mathrm{~mm} ; \mathrm{AL}=0.54-0.56 \mathrm{~mm} ; \mathrm{AW}=0.76-0.79 \mathrm{~mm}$; $\mathrm{TBL}=2.29-2.42 \mathrm{~mm} ; \mathrm{EL}=0.71-0.76 \mathrm{~mm} ; \mathrm{EW}=0.85-0.89 \mathrm{~mm} ; \mathrm{HL}=0.50-0.53 \mathrm{~mm} ; \mathrm{HW}=0.52$ $0.55 \mathrm{~mm} ; \mathrm{LE}=0.14 \mathrm{~mm} ; \mathrm{LT}=0.17 \mathrm{~mm} ; \mathrm{PL}=0.54-0.57 \mathrm{~mm} ; \mathrm{PW}=0.53-0.56 \mathrm{~mm}$.

Body (Fig. 14A) reddish-brown, mouthparts and tarsi paler. Head approximately as long as broad. Each eye composed of about 20 facets; $\mathrm{LE}: \mathrm{LT}=0.8$. Antennal clubs (Fig. 14B) formed by antennomeres 9-11; antennomere 9 (Fig. 14C) strongly transverse when widths are fully visible, each with semi-membranous 
apophysis at apical $2 / 5$ of mesal margin; antennomere 10 strongly transverse, obconical; antennomere 11 (Fig. 14B) about 1.2 times as long as wide, each with single broad basal protuberance covered with tuft of setae at apex (Fig. 14D). Pronotum about as long as wide. Elytra moderately transverse. Abdomen widest at tergite 1 (IV) and narrowing apically. Aedeagus (Fig. 14E) moderately asymmetrical.

\section{Female}

Unknown.

\section{Distribution}

China: Guangxi.

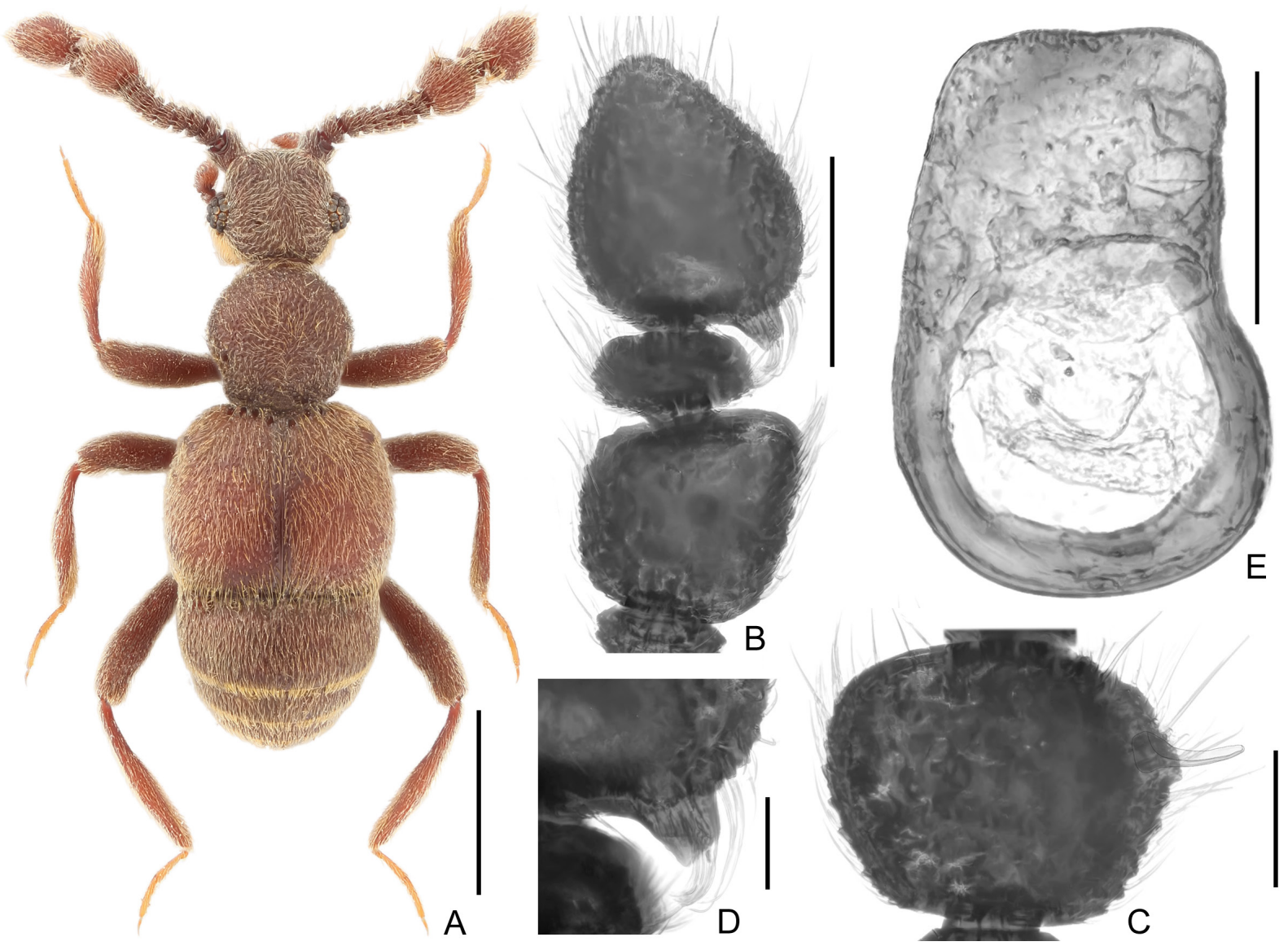

Fig. 14. Sathytes valentulus sp. nov., $\hat{O}$ (population of Laoshan Forest Farm). A. Dorsal habitus. B. Antennal club. C. Antennomere 9. D. Modification of antennomere 11, enlarged. E. Aedeagus, ventral view. Scale bars: $\mathrm{A}=0.5 \mathrm{~mm} ; \mathrm{B}=0.2 \mathrm{~mm} ; \mathrm{C}, \mathrm{E}=0.1 \mathrm{~mm} ; \mathrm{D}=0.05 \mathrm{~mm}$. 
Sathytes xingdoumontis sp. nov.

urn:1sid:zoobank.org:act:F8A011D4-D284-410E-A1BA-EF0A72463BC7

Fig. 15

\section{Differential diagnosis}

Among the species possessing a single basal protuberance of antennomere 11 and a conspicuously oblique mesal margin of antennomere 9 (see Differential diagnosis under S. shennong sp. nov.), the male of S. xingdoumontis sp. nov. can be readily distinguished by the unique position and shape of the apophysis of antennomere 9 .

\section{Etymology}

The new species is named after Xingdoushan Nature Reserve, where the type locality of this species is situated.
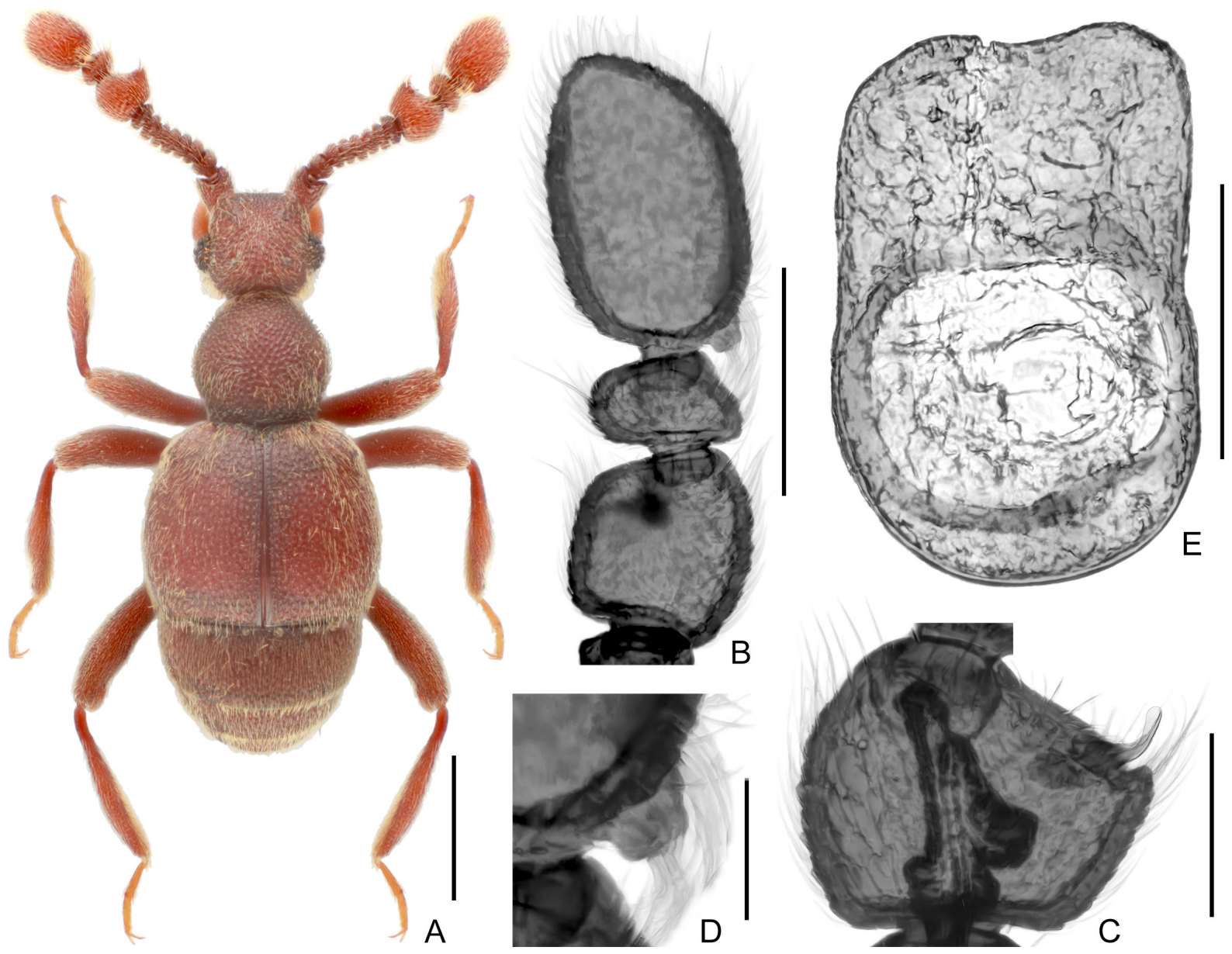

Fig. 15. Sathytes xingdoumontis sp. nov., §̂. A. Dorsal habitus. B. Antennal club. C. Antennomere 9. D. Modification of antennomere 11, enlarged. E. Aedeagus, ventral view. Scale bars: $A=0.5 \mathrm{~mm} ; \mathrm{B}=$ $0.2 \mathrm{~mm} ; \mathrm{C}, \mathrm{E}=0.1 \mathrm{~mm} ; \mathrm{D}=0.05 \mathrm{~mm}$. 
Type material (2 specimens)

\section{Holotype}

CHINA - ô; Hubei, Enshi (恩施), Xingdoushan N.R. (星斗山自然保护区), San-Xian-Chang (三县场); 30²'20.48" N, 1098'33.89" E; alt. 1114 m; 19 May 2017; Zhou G.C., Tian T. and Huang Z.G. leg.; sifted; SNUC.

\section{Paratype}

CHINA • 1 क ; same collection data as for holotype; $30^{\circ} 2^{\prime} 29.98^{\prime \prime} \mathrm{N}, 109^{\circ} 8^{\prime} 1.60^{\prime \prime} \mathrm{E}$; alt. $1253 \mathrm{~m}$; 18 May 2017; SNUC.

\section{Description}

\section{Male}

Measurements. AeL $=0.32 \mathrm{~mm} ; \mathrm{AnL}=1.20 \mathrm{~mm} ; \mathrm{AL}=0.51 \mathrm{~mm} ; \mathrm{AW}=0.69 \mathrm{~mm} ; \mathrm{TBL}=2.15 \mathrm{~mm}$; $\mathrm{EL}=0.69 \mathrm{~mm} ; \mathrm{EW}=0.84 \mathrm{~mm} ; \mathrm{HL}=0.46 \mathrm{~mm} ; \mathrm{HW}=0.46 \mathrm{~mm} ; \mathrm{LE}=0.16 \mathrm{~mm} ; \mathrm{LT}=0.13 \mathrm{~mm} ; \mathrm{PL}=$ $0.49 \mathrm{~mm} ; \mathrm{PW}=0.48 \mathrm{~mm}$.

Body (Fig. 15A) reddish-brown, mouthparts and tarsi paler. Head about as long as wide. Each eye composed of about 22 facets; LE:LT = 1.2. Antennal clubs (Fig. 15B) formed by antennomeres 9-11; antennomere 9 (Fig. 15C) angularly expanded mesally, each with semi-membranous apophysis anterior to apex of expansion; antennomere 10 transverse, obconical; antennomere 11 (Fig. 15B) about 1.5 times as long as wide, each with single short basal protuberance covered with tuft of setae at apex (Fig. 15D). Pronotum approximately as long as wide. Elytra moderately transverse. Abdomen widest at tergite 1 (IV) and narrowing apically. Aedeagus (Fig. 15E) lobe slightly constricted near middle, almost symmetrical.

\section{Female}

Measurements. $\mathrm{AnL}=0.97 \mathrm{~mm} ; \mathrm{AL}=0.69 \mathrm{~mm} ; \mathrm{AW}=0.75 \mathrm{~mm} ; \mathrm{TBL}=2.21 \mathrm{~mm} ; \mathrm{EL}=0.52 \mathrm{~mm}$; $\mathrm{EW}=0.51 \mathrm{~mm} ; \mathrm{HL}=0.48 \mathrm{~mm} ; \mathrm{HW}=0.48 \mathrm{~mm} ; \mathrm{LE}=0.15 \mathrm{~mm} ; \mathrm{LT}=0.16 \mathrm{~mm} ; \mathrm{PL}=0.52 \mathrm{~mm} ; \mathrm{PW}=$ $0.51 \mathrm{~mm}$.

Slightly larger than male in size. Each eye composed of about 14 facets; LE: LT $=0.9$. Antennal clubs lacking modifications.

\section{Distribution}

China: Hubei.

Sathytes xizangensis sp. nov.

urn:1sid:zoobank.org:act:F9F70319-A210-4FAC-A99E-1176460E4E55

Fig. 16

\section{Differential diagnosis}

The male of S. xizangensis sp. nov. is morphologically similar to that of S. grandis (Nepal), S. chayuensis sp. nov. (China: Xizang), S. magnus (China: Xizang) and S. tangliangi (China: Yunnan) in sharing the single basal protuberance of antennomere 11 and oblique mesal margin of antennomere 9 . It can be separated from $S$. grandis and $S$. magnus by the much smaller body size $(2.53-2.73 \mathrm{~mm}$ vs $3 \mathrm{~mm}$ for S. grandis and $3.36 \mathrm{~mm}$ for $S$. magnus) and more distal position of the apophysis of antennomere 9; from S. chayuensis sp. nov. by the much shorter antennomere 11; and from S. tangliangi by the larger basal protuberance and more distal position of the apophysis of antennomere 9. 


\section{Etymology}

The new species is named after Xizang Province (= Tibet), where the type locality of this species is situated.

\section{Type material (29 specimens)}

\section{Holotype}

CHINA • ${ }^{\wedge}$; Xizang, Nyingchi, Pailong Country; 300'34.71" N, 9457'57.64" E; alt. 2190 m; 15 Jul.1 Aug. 2019; Z.-W. Yin leg.; FIT (flight intercept trap); SNUC.

\section{Paratypes}

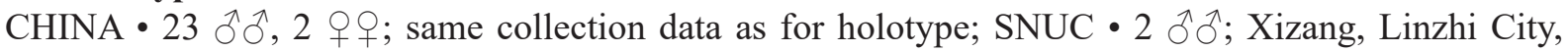
Pailong Village; alt. $2069 \mathrm{~m}, 30^{\circ} 01^{\prime} 11.11^{\prime \prime}$ N, 9500'0.46" E; 15 Mar. 2017; X.-B. Song leg.; SNUC

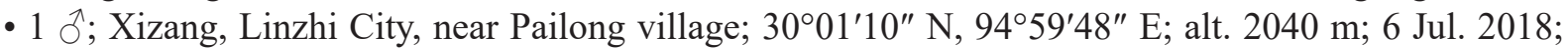
Cheng, Peng and Shen leg.; mixed leaf litter, sifted; SNUC.

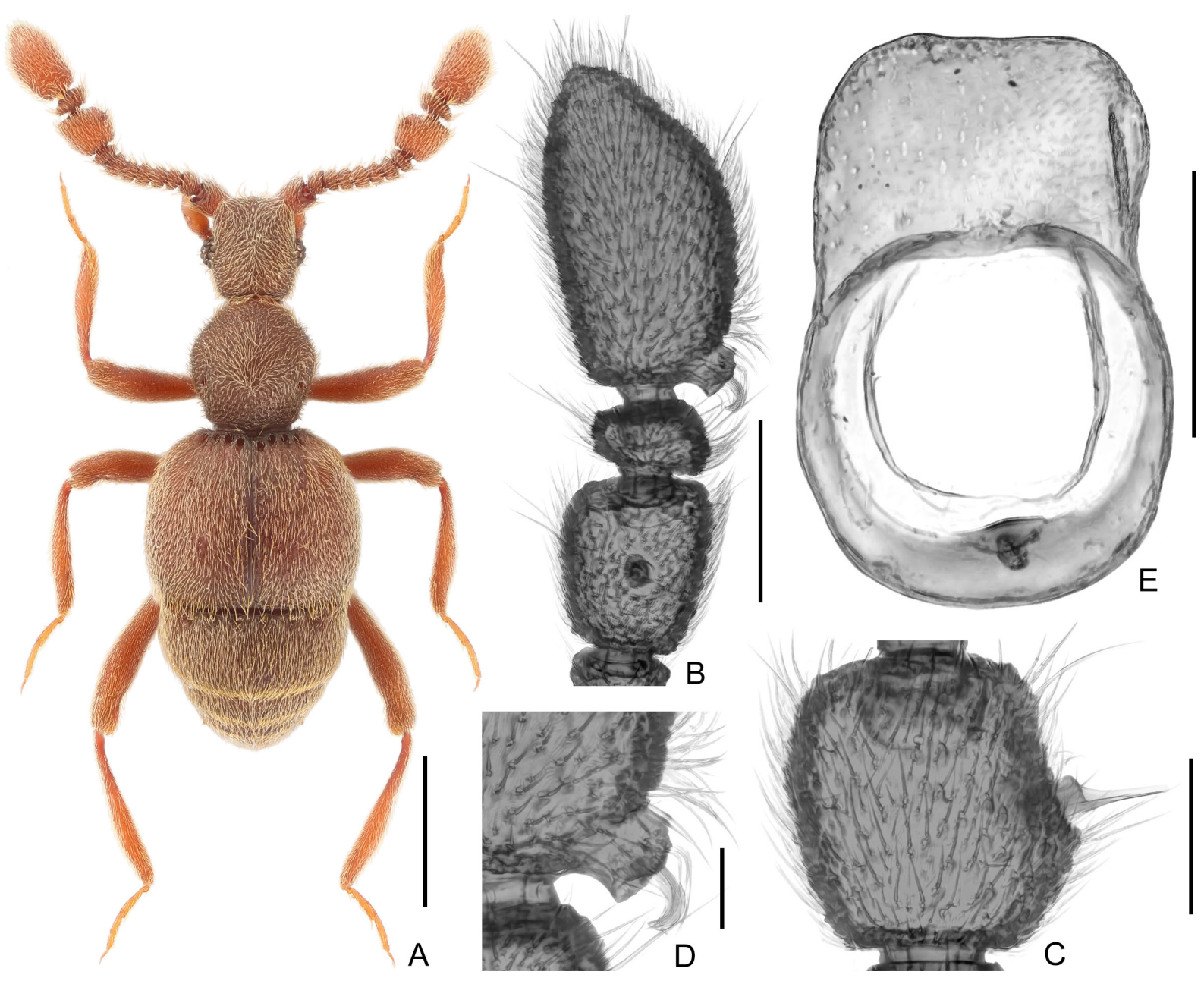

Fig. 16. Sathytes xizangensis sp. nov., $\widehat{\partial}$ (population of Pailong). A. Dorsal habitus. B. Antennal club. C. Antennomere 9. D. Modification of antennomere 11, enlarged. E. Aedeagus, ventral view. Scale bars: $\mathrm{A}=0.5 \mathrm{~mm} ; \mathrm{B}=0.2 \mathrm{~mm} ; \mathrm{C}, \mathrm{E}=0.1 \mathrm{~mm} ; \mathrm{D}=0.05 \mathrm{~mm}$. 


\title{
Description
}

Male

MeAsurements. AeL $=0.22 \mathrm{~mm} ; \mathrm{AnL}=1.25-1.29 \mathrm{~mm} ; \mathrm{AL}=0.65-0.73 \mathrm{~mm} ; \mathrm{AW}=0.82-0.88 \mathrm{~mm}$; $\mathrm{TBL}=2.53-2.73 \mathrm{~mm} ; \mathrm{EL}=0.76-0.83 \mathrm{~mm} ; \mathrm{EW}=0.95-1.01 \mathrm{~mm} ; \mathrm{HL}=0.55-0.58 \mathrm{~mm} ; \mathrm{HW}=0.46-$ $0.49 \mathrm{~mm} ; \mathrm{LE}=0.17 \mathrm{~mm} ; \mathrm{LT}=0.17 \mathrm{~mm} ; \mathrm{PL}=0.57-0.59 \mathrm{~mm} ; \mathrm{PW}=0.55-0.57 \mathrm{~mm}$.

Body (Fig. 16A) reddish-brown, mouthparts and tarsi paler. Head longer than wide. Each eye composed of about 22 facets; LE: LT = 1. Antennal clubs (Fig. 16B) formed by antennomeres 9-11; inner margin of antennomere 9 (Fig. 16C) moderately oblique, semi-membranous apophysis at middle of mesal margin; antennomere 10 transverse, obconical; antennomere 11 (Fig. 16B) about 1.7 times as long as wide, each with single basal protuberance covered with tuft of setae at apex (Fig. 16D). Pronotum approximately as long as broad. Elytra moderately transverse. Abdomen widest at tergite 1 (IV) and narrowing apically. Aedeagus (Fig. 16E) almost symmetrical.

\section{Female}

Measurements. AnL $=1.17-1.21 \mathrm{~mm} ; \mathrm{AL}=0.77-0.78 \mathrm{~mm} ; \mathrm{AW}=0.78-0.79 \mathrm{~mm} ; \mathrm{TBL}=2.58-2.67 \mathrm{~mm}$; $\mathrm{EL}=0.71-0.73 \mathrm{~mm} ; \mathrm{EW}=0.88-0.90 \mathrm{~mm} ; \mathrm{HL}=0.54-0.58 \mathrm{~mm} ; \mathrm{HW}=0.46-0.49 \mathrm{~mm} ; \mathrm{LE}=0.15 \mathrm{~mm}$; $\mathrm{LT}=0.17 \mathrm{~mm} ; \mathrm{PL}=0.56-0.58 \mathrm{~mm} ; \mathrm{PW}=0.54-0.56 \mathrm{~mm}$.

Similar to male in size. Each eye composed of about 20 facets; LE:LT =0.9. Antennal clubs lacking modifications.

\section{Distribution}

China: Xizang.

Sathytes yunnanicus Yin \& Li, 2012

Sathytes yunnanicus Yin \& Li in Yin et al. 2012: 853, figs 3o, 6c, j, 7o, 8 o.

Material examined (3 specimens)

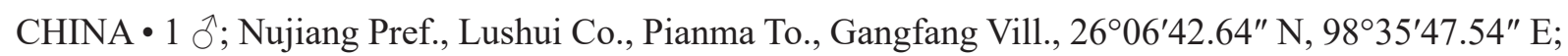

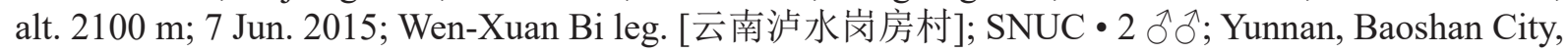
Tengchong County, Mingguang Town, Baihua Ling; $25^{\circ} 18^{\prime} 10^{\prime \prime}$ N, 98 $98^{\circ} 7^{\prime} 31^{\prime \prime}$ E; alt. 1870 m; 21 Apr. 2013; Dai, Song and Peng leg.; SNUC.

\section{Remarks}

This species was originally described based on a single male from Qiqi, Gongshan County. Additional specimens show that it has a wider distribution in Yunnan.

\section{Distribution}

China: Yunnan.

\section{Checklist of world species (updated from Yin et al. 2012)}

\author{
Genus Sathytes Westwood, 1870
}

(57 spp. in Palaearctic and Oriental regions)

Sathytes Westwood, 1870: 128. Type species: S. punctiger Westwood, 1870 (by original monotypy).

Batoxylina Jeannel, 1957: 8 (syn. by Löbl 1979: 285). Type species: Batoxylina clavalis Jeannel, 1957

(by original designation). 
1. S. alpicola Yin \& Shen sp. nov. China: Xizang.

2. S. appendiculatus Löbl, 1979: 292. India: Darjeeling; Nepal: Pokhara.

3. S. australis Yin \& Shen sp. nov. China: Guangdong, Guangxi.

4. S. borneoensis Shen \& Yin, 2019: 189. East Malaysia: Sabah.

5. S. chayuensis Yin \& Shen sp. nov. China: Xizang.

6. S. chengzhifeii Yin \& Shen sp. nov. China: Yunnan.

7. S. clavalis (Jeannel, 1957: 9). Vietnam: Hòa Bình.

Batoxylina clavalis Jeannel, 1957: 9.

Sathytes clavalis - Löbl 1979: 293.

8. S. clavatus Löbl, 1979: 295. Indonesia: Sumatra.

9. S. communis Shen \& Yin, 2020: 387. China: Anhui, Zhejiang, Jiangxi.

10. S. cristatus Yin \& Li, 2012 (in Yin et al. 2012: 835). China: Yunnan.

11. S. excavatus Löbl, 1979: 291. Nepal: Pokhara.

12. S. excertus Yin \& Li, 2012 (in Yin et al. 2012: 837). China: Yunnan.

13. S. fenghuang Shen \& Yin, 2020: 388. China: Zhejiang.

14. S. franzi Löbl, 1979: 292. Nepal: Kali-Gandaki.

15. S. fuliginosus (Jeannel, 1960: 208). India: Darjeeling.

Batoxyla fuliginosa Jeannel, 1960: 208

Sathytes fuliginosus - Löbl 1979: 302.

16. S. grandis Löbl, 1979: 298. Napel: Trisuli Bazar.

17. S. hamulifer Löbl, 1979: 296. Nepal: Kali-Gandaki.

18. S. huapingensis Yin \& Shen sp. nov. China: Guangxi.

19. S. imitator Löbl, 1979: 291. Nepal: Katmandu.

20. S. jinggang Shen \& Yin, 2020: 389. China: Jiangxi.

21. S. larifuga Shen \& Yin, 2019: 191. East Malaysia: Sabah.

22. S. larinus Yin \& Li, 2012 (in Yin et al. 2012: 842). China: Zhejiang, Fujian.

23. S. linzhiensis Yin \& Shen sp. nov. China: Xizang.

24. S. liuyei Shen \& Yin, 2019: 190. East Malaysia: Sabah.

25. S. longitrabis Yin \& Li, 2012 (in Yin et al. 2012: 843). China: Shaanxi.

26. S. longwangshanus Yin \& Li, 2012 (in Yin et al. 2012: 844). China: Zhejiang.

27. S. magnus Yin \& Li, 2012 (in Yin et al. 2012: 845). China: Xizang.

28. S. maoershanus Yin \& Shen sp. nov. China: Guangxi.

29. S. minutus Löbl, 1979: 301. Nepal: Godavari.

30. S. montanus Löbl, 1979: 297. India: Chopal-Khangna Nallah.

31. S. nujiangensis Yin \& Shen sp. nov. China: Yunnan.

32. S. obliquus Shen \& Yin, 2020: 392. China: Zhejiang.

33. S. oculatus Löbl, 1979: 296. Indonesia: Java.

34. S. paulus Yin \& Li, 2012 (in Yin et al. 2012: 846). China: Zhejiang.

35. S. panzhaohuii Yin \& Shen sp. nov. China: Xizang.

36. S. perpusillus Yin \& Li, 2012 (in Yin et al. 2012: 847). China: Yunnan.

37. S. punctiger Westwood, 1870: 128. East Malaysia: Sarawak.

38. S. rarus Yin \& Li, 2012 (in Yin et al. 2012: 848). China: Yunnan.

39. S. reductus Löbl, 1979: 301. Indonesia: Sumatra.

40. S. rufus Raffray, 1894: 281. Indonesia: Sumatra; West Malaysia: Penang.

S. gracilis Raffray, 1904: 65 (syn. by Löbl 1979: 287).

41. S. shennong Yin \& Shen sp. nov. China: Hubei.

42. S. shihongliangi Shen \& Yin, 2019: 192. East Malaysia: Sabah.

43. S. sichuanicus Yin \& Li, 2012 (in Yin et al. 2012: 849). China: Sichuan.

44. S. similis Shen \& Yin, 2020: 394. China: Zhejiang, Jiangxi.

45. S. simplex Löbl, 1979: 298. India: Darjeeling. 
46. S. tangliangi Yin \& Li, 2012 (in Yin et al. 2012: 850). China: Yunnan.

47. S. tianquanus Yin \& Shen sp. nov. China: Sichuan.

48. S. tibialis Yin \& Li, 2012 (in Yin et al. 2012: 850). China: Yunnan.

49. S. transversus Yin \& Shen sp. nov. China: Xizang.

50. S. usitatus Yin \& Li, 2012 (in Yin et al. 2012: 851). China: Yunnan.

51. S. vafelus Löbl, 1979: 289. Nepal: Pokhara.

52. S. valentulus Yin \& Shen sp. nov. China: Guangxi.

53. S. vespertinus Raffray, 1890: 204. West Malaysia: Penang.

54. S. wuyishanus Yin \& Li, 2012 (in Yin et al. 2012: 852). China: Fujian.

55. S. xingdoumontis Yin \& Shen sp. nov. China: Hubei.

56. S. xizangensis Yin \& Shen sp. nov. China: Xizang.

57. S. yunnanicus Yin \& Li, 2012 (in Yin et al. 2012: 853). China: Yunnan.

\section{Discussion}

Correct species identifications of Sathytes require careful examinations of the form of the male antennal clubs, assisted by body size and geographical distribution. Although a number of species can be readily diagnosed and distinguished by one or two characters alone, e.g., S. chayuensis sp. nov. has a conspicuously elongate antennomere 11, and S. appendiculatus Löbl, 1979 has the basal protuberance of antennomere 11 much longer than the subbasal protuberance, the majority of the species are morphologically similar to one or more congeners, and usually can be separated only by subtle differences, such as different proportions of antennomeres 9 and 11, the location and shape of the apophysis on antennomere 9, etc. Thus, as the number of species grows rapidly, a direct comparison to the holotype or identified specimens of known species may be frequently needed for establishing new taxa. For the same reason, an identification key to the Chinese or world species, which is difficult to make and considered of little use in identification, is not provided here.

The distribution of Sathytes in China is shown in Figs 17-22. The range goes from Zhejiang in the east to Xizang in the west, and from Shaanxi in the north to southern Yunnan in the south, with many provinces/areas still lacking proper sampling (Fig. 17A). Since many species of Sathytes are known only from the type locality, or described based on a very limited number of specimens (Figs 17B-E, 18-22), future collection efforts in poorly sampled areas are needed to reveal the true diversity of the genus. Such efforts would likely lead to the discovery of a great number of undescribed species, as evidenced by the discovery of 15 new species in the present paper. 

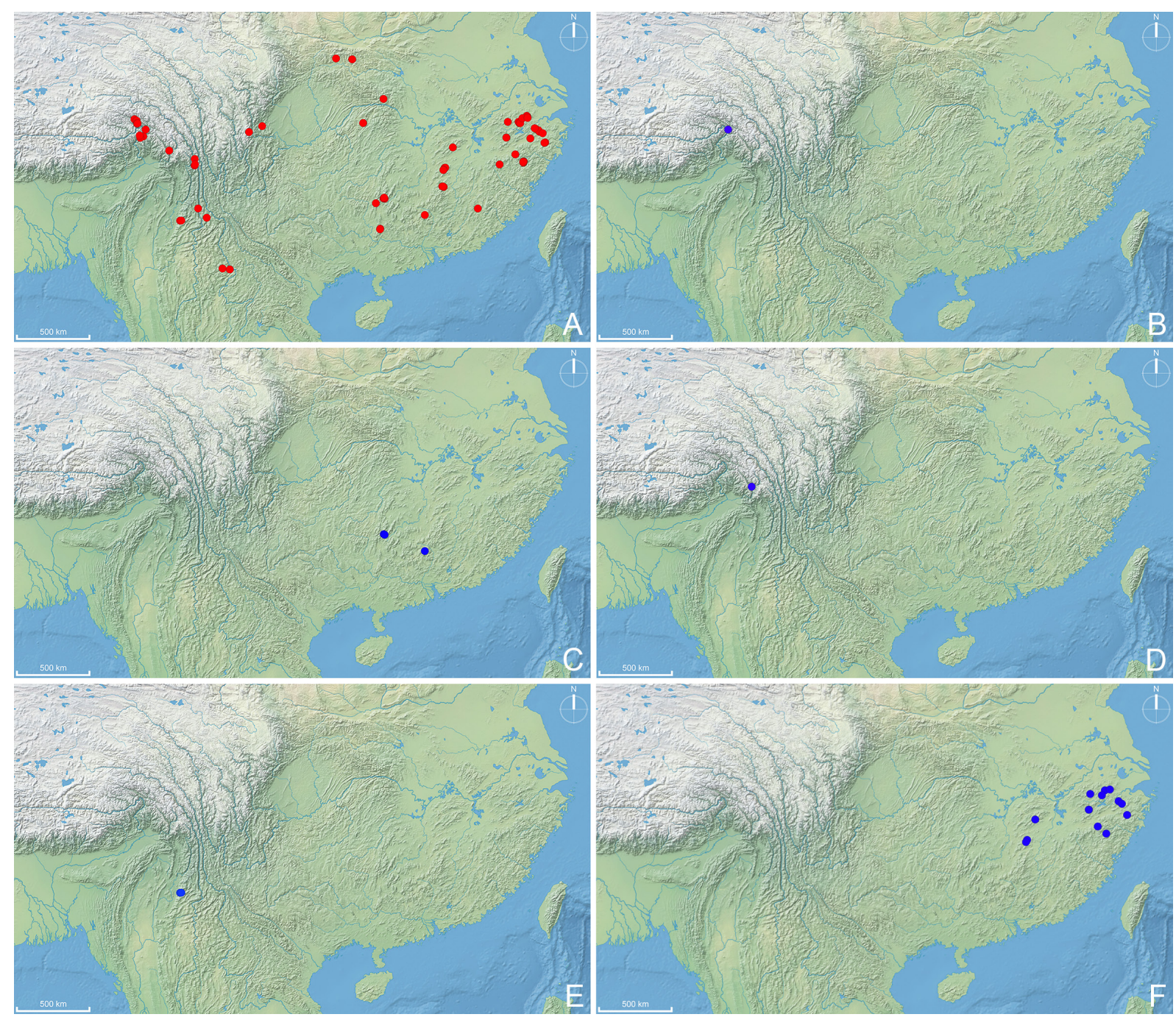

Fig. 17. Distribution of Sathytes Westwood, 1870 in China. A. General distribution. B. S. alpicola sp. nov. C. S. australis sp. nov. D. S. chayuensis sp. nov. E. S. chengzhifeii sp. nov. F. S. communis Shen \& Yin, 2020. 

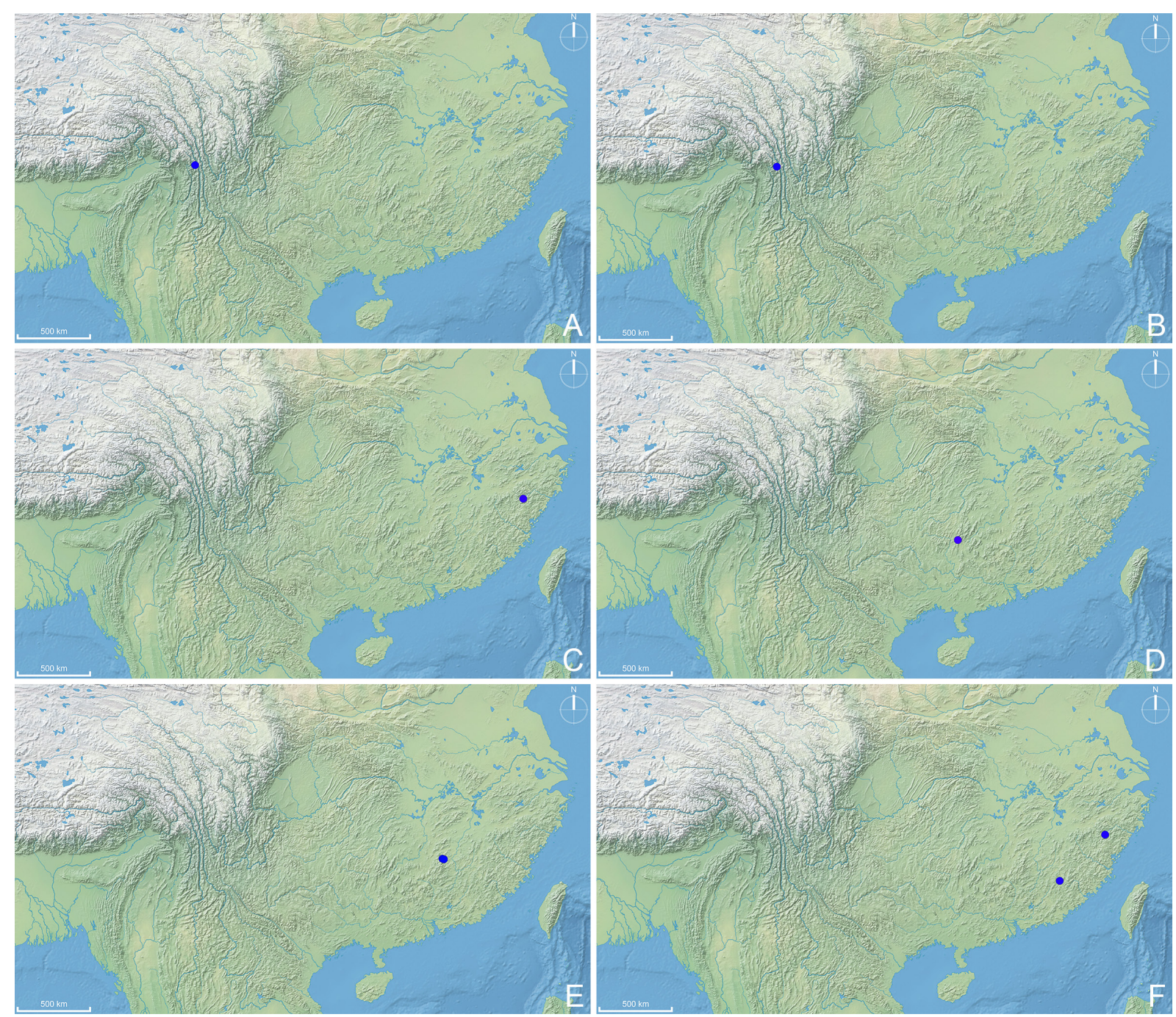

Fig. 18. Distribution of Sathytes Westwood, 1870 in China. A. S. cristatus Yin \& Li, 2012. B. S. excertus Yin \& Li, 2012. C. S. fenghuang Shen \& Yin, 2020. D. S. huapingensis sp. nov. E. S. jinggang Shen \& Yin, 2020. F. S. larinus Yin \& Li, 2012. 

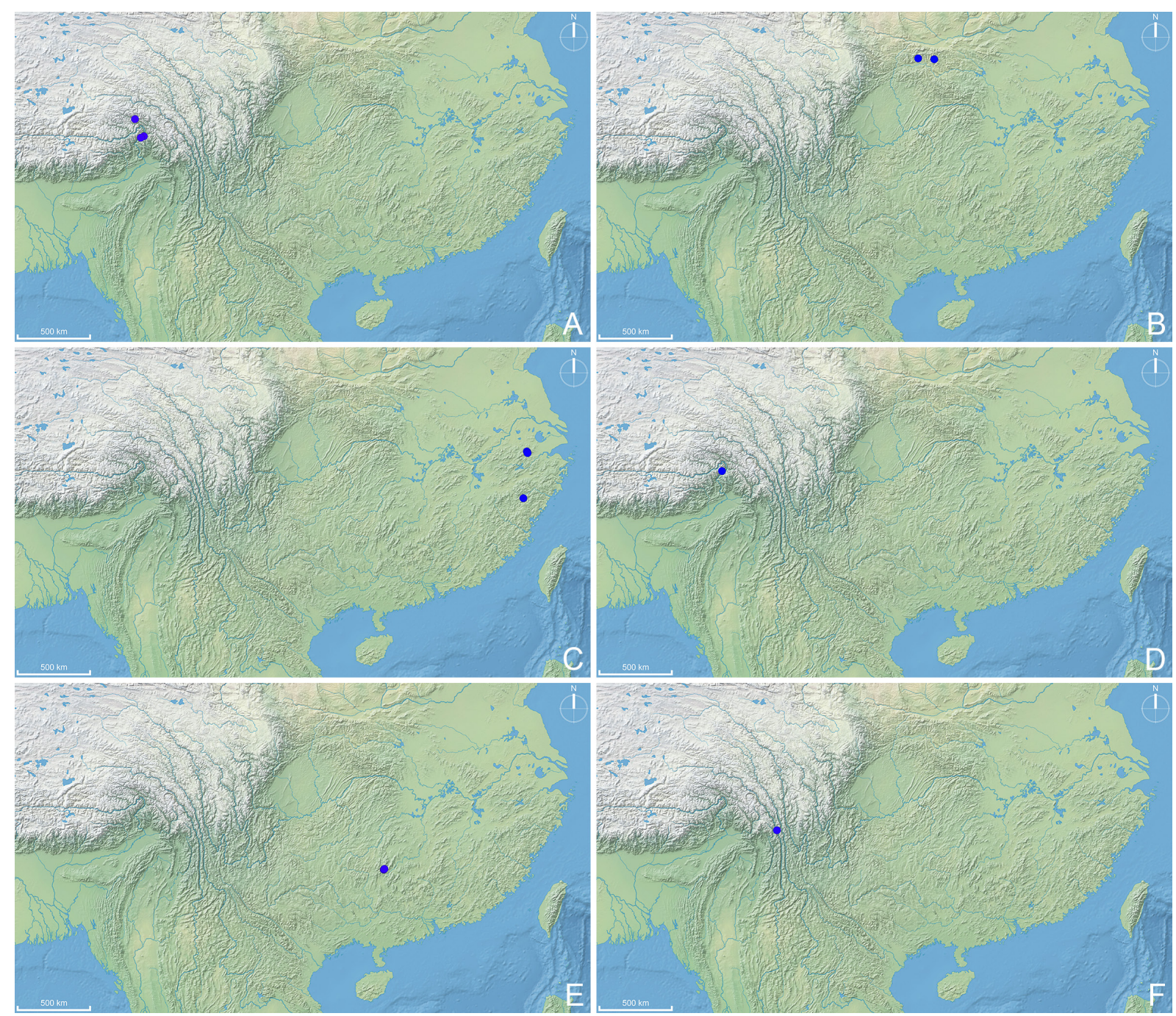

Fig. 19. Distribution of Sathytes Westwood, 1870 in China. A. S. linzhiensis sp. nov. B. S. longitrabis Yin \& Li, 2012. C. S. longwangshanus Yin \& Li, 2012. D. S. magnus Yin \& Li, 2012. E. S. maoershanus sp. nov. F. S. nujiangensis sp. nov. 

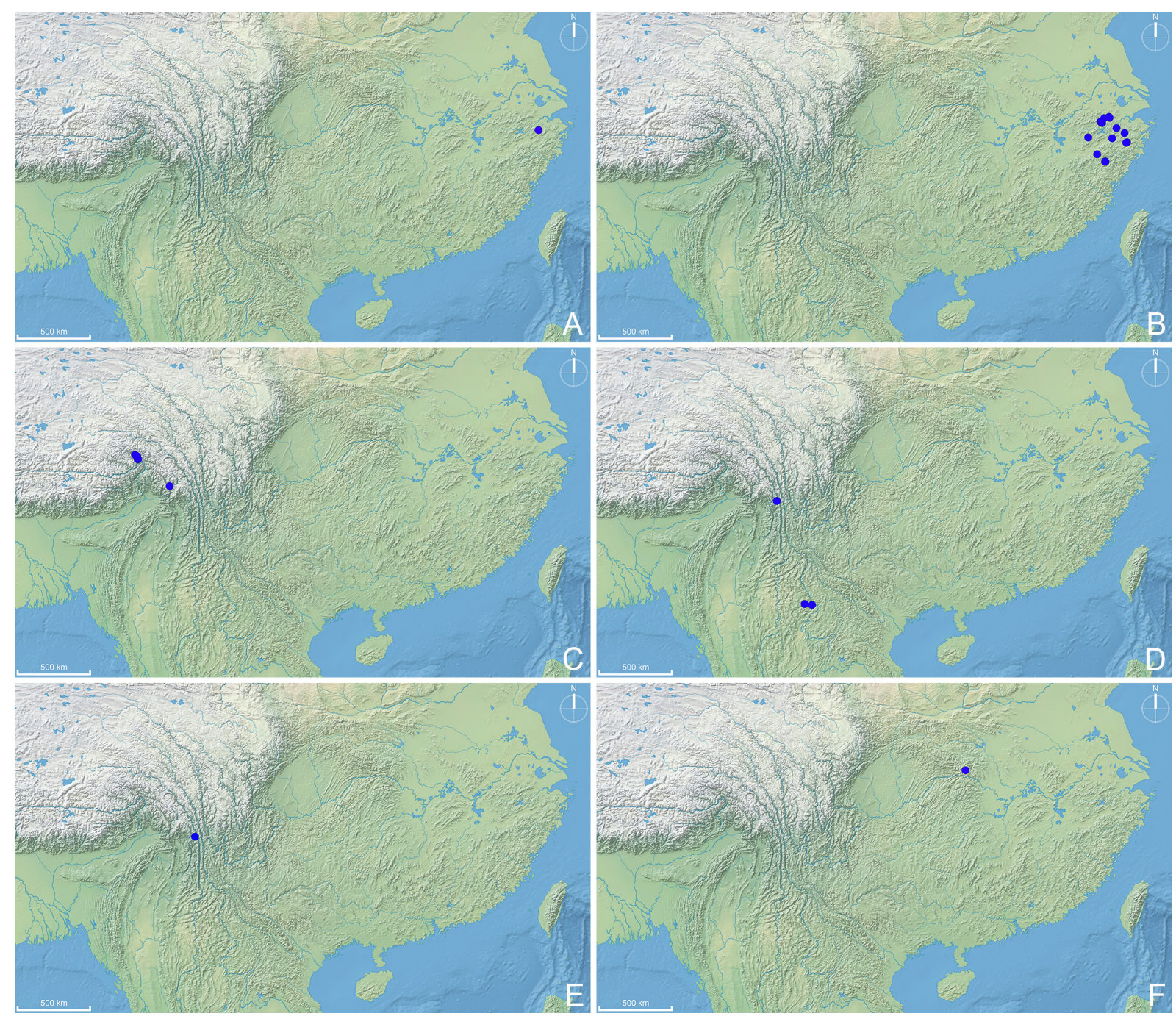

Fig. 20. Distribution of Sathytes Westwood, 1870 in China. A. S. obliquus Shen \& Yin, 2020. B. S. paulus Yin \& Li, 2012. C. S. panzhaohuii sp. nov. D. S. perpusillus Yin \& Li, 2012. E. S. rarus Yin \& Li, 2012. F. S. shennong sp. nov. 

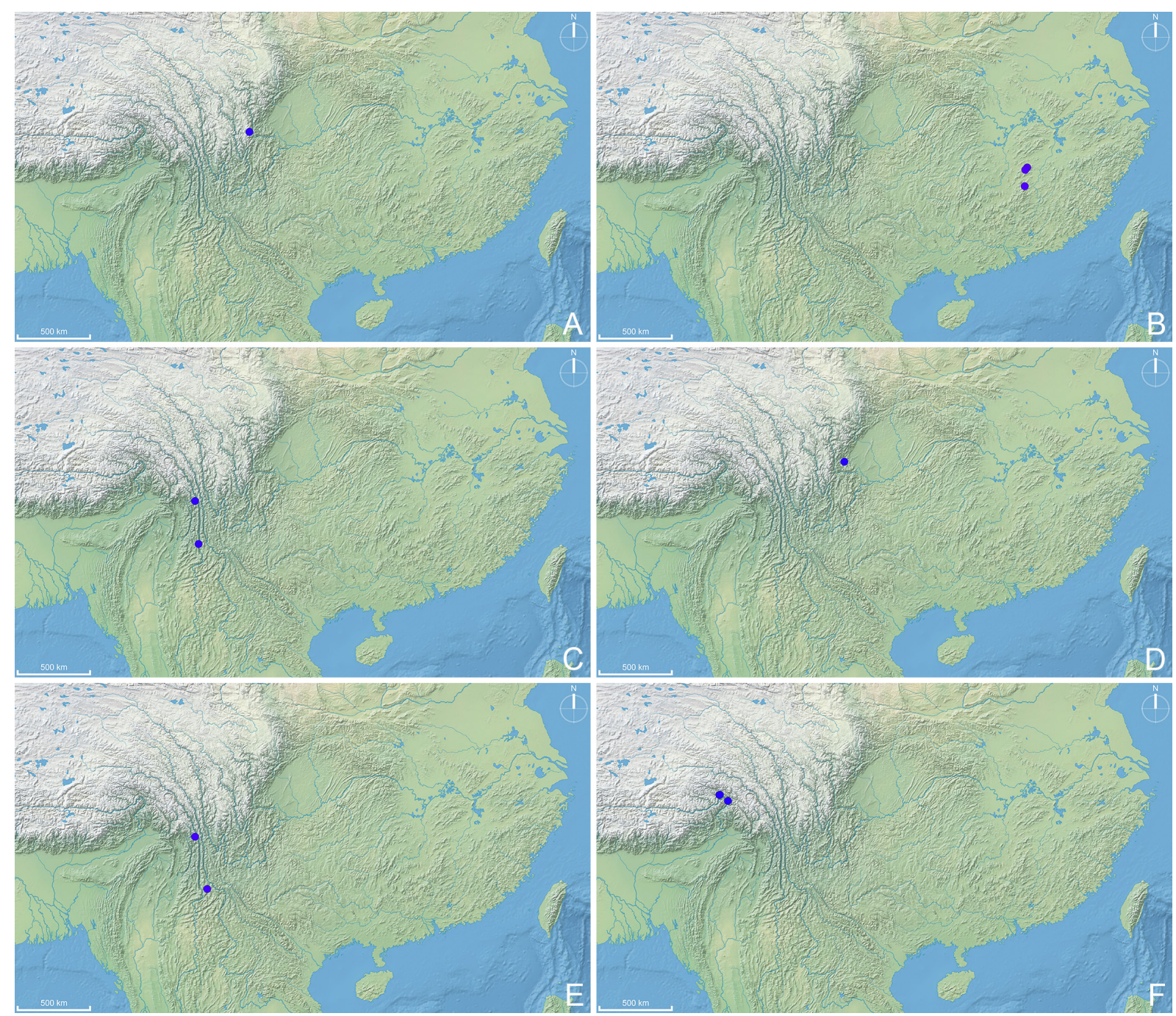

Fig. 21. Distribution of Sathytes Westwood, 1870 in China. A. S. sichuanicus Yin \& Li, 2012. B. S. similis Shen \& Yin, 2020. C. S. tangliangi Yin \& Li, 2012. D. S. tianquanus sp. nov. E. S. tibialis Yin \& Li, 2012. F. S. transversus sp. nov. 

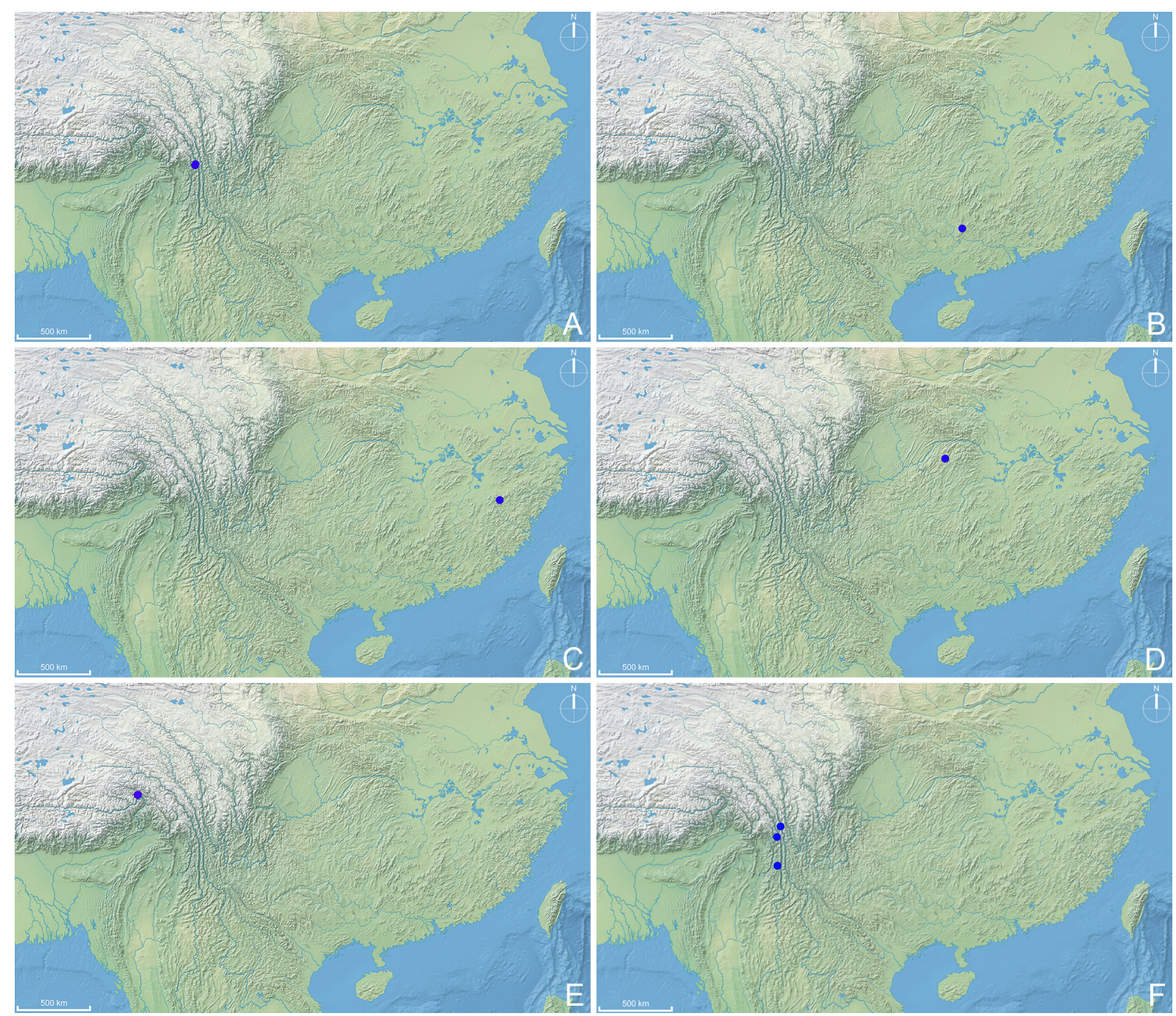

Fig. 22. Distribution of Sathytes Westwood, 1870 in China. A. S. usitatus Yin \& Li, 2012. B. S. valentulus sp. nov. C. S. wuyishanus Yin \& Li, 2012. D. S. xingdoumontis sp. nov. E. S. xizangensis sp. nov. F. S. yunnanicus Yin \& Li, 2012. 


\section{Acknowledgements}

Two anonymous reviewers provided very helpful comments on the draft manuscript, which considerably improved the paper. The present study was supported by the National Natural Science Foundation of China (No. 31872965), and the 'Phosphorus' Science Foundation, Shanghai Municipal Science and Technology Commission, China (19QA1406600) awarded to ZWY.

\section{References}

Jeannel R. 1957. Sur quelques Psélaphides du Tonkin recueillis par le Père A. de Cooman. Revue Française d'Entomologie 24: 5-32.

Jeannel R. 1960. Sur les Psélaphides (Coleoptera) de l'Inde septentrionale. Bulletin of the British Museum (Natural History). Entomology 9: 401-456. https://doi.org/10.5962/bhl.part.27558

Löbl I. 1979. Révision du genre Sathytes Westwood (Coleoptera, Pselaphidae). Revue suisse de Zoologie 86: 285-307. https://doi.org/10.5962/bhl.part.82294

Raffray A. 1890. Étude sur les Psélaphides. VI. Diagnoses des espèces nouvelles sur lesquelles sont fondés des genres nouveaux. Revue d'Entomologie 9: 193-219.

Raffray A. 1894. Révision des Psélaphides des îles de Singapore et de Penang. Revue d'Entomologie 13: 197-282.

Raffray A. 1904. Genera et catalogue des Psélaphides. Annales de la Société entomologique de France 73: $1-476$.

Shen Q. \& Yin Z.-W. 2019. Four new species of Sathytes Westwood (Coleoptera: Staphylinidae: Pselaphinae) from the island of Borneo. Zootaxa 4629: 188-194.

https://doi.org/10.11646/zootaxa.4629.2.2

Shen Q. \& Yin Z.-W. 2020. Five new species and a new record of Sathytes Westwood from eastern China (Coleoptera: Staphylinidae: Pselaphinae). Zootaxa 4751: 386-394.

https://doi.org/10.11646/zootaxa.4751.2.13

Yin Z.-W., Li L.-Z. \& Zhao M.-J. 2012. Taxonomic study on Sathytes Westwood (Coleoptera: Staphylinidae: Pselaphinae) from China. Part I. Journal of Natural History 46: 831-857.

https://doi.org/10.1080/00222933.2011.651651

Westwood J.O. 1870. Descriptions of twelve new exotic species of the Coleopterous family Pselaphidae. Transactions of the Royal Entomological Society of London 1870: 125-132.

https://doi.org/10.1111/j.1365-2311.1870.tb01868.x

Manuscript received: 3 July 2020

Manuscript accepted: 16 September 2020

Published on: 26 October 2020

Topic editor: Nesrine Akkari

Section editor: Max Barclay

Desk editor: Radka Rosenbaumová

Printed versions of all papers are also deposited in the libraries of the institutes that are members of the EJT consortium: Muséum national d'histoire naturelle, Paris, France; Meise Botanic Garden, Belgium; Royal Museum for Central Africa, Tervuren, Belgium; Royal Belgian Institute of Natural Sciences, Brussels, Belgium; Natural History Museum of Denmark, Copenhagen, Denmark; Naturalis Biodiversity Center, Leiden, the Netherlands; Museo Nacional de Ciencias Naturales-CSIC, Madrid, Spain; Real Jardín Botánico de Madrid CSIC, Spain; Zoological Research Museum Alexander Koenig, Bonn, Germany; National Museum, Prague, Czech Republic. 